\title{
Collapsed, uncollapsed, and hidden magnetic flux on the quiet Sun ${ }^{\star}$
}

\author{
J. O. Stenflo ${ }^{1,2}$ \\ ${ }^{1}$ Institute of Astronomy, ETH Zurich, 8093 Zurich, Switzeland \\ e-mail: stenflo@astro.phys.ethz.ch \\ 2 Istituto Ricerche Solari Locarno, via Patocchi, 6605 Locarno Monti, Switzerland
}

Received 6 December 2010 / Accepted 18 February 2011

\section{ABSTRACT}

\begin{abstract}
Since the first applications of the Stokes $V$ line ratio in the early 1970s and the Hanle depolarization effect in the early 1980 s we have had a dualistic view of quiet-Sun magnetism: intermittent $\mathrm{kG}$ flux tubes surrounded by an ocean of turbulent fields with strengths of order 10-100 G. There has been the concern that this dualism could be an artefact of using two mutually almost exclusive diagnostic tools, the Zeeman and Hanle effect. We find however that the Hinode line-ratio data alone, without any reference to the Hanle effect, reveal the existence of two distinct flux populations, representing strong (collapsed) and weak (uncollapsed) flux. The collapsed population is preferentially located in the intergranular lanes, while the uncollapsed population is most visible in the bright cell interiors. From a comparison between the intrinsic field strengths, as derived from the line ratio for the collapsed population, and the corresponding flux densities, we can deduce the size distribution of the flux tubes. The majority of them are found to have sizes in the range 10-70 km. The intrinsic flux tube field strength decreases with diminishing size to become substantially smaller than kG for sizes below about $60 \mathrm{~km}$. Comparison between the average of the unsigned flux density in the Hinode quiet-Sun data set and earlier constraints from the Hanle depolarization effect shows that most of the flux remains invisible at the Hinode resolution scale due to cancellation of the opposite magnetic polarities within the spatial resolution element. We have derived the cancellation function that describes how the visibility of the hidden flux improves with increased spatial resolution. It needs to be extrapolated to extremely small scales before the constraints imposed by the Hanle effect get satified, which suggests that the bulk of the hidden flux resides at scales near the end of the magnetic scale spectrum (of order $10 \mathrm{~m}$ ).
\end{abstract}

Key words. Sun: atmosphere - magnetic fields - polarization - magnetohydrodynamics (MHD)

\section{Introduction}

It has long been recognized that the key to understanding solar magnetism lies in the scales that are far smaller than the telescope resolution. Forty years ago two fundamental concepts to address and diagnose the magnetic properties in the spatially unresolved domain were introduced: (1) the concept of a 2component model to diagnose the intermittent nature of solar magnetic fields, and (2) the concept of a tangled, micro-turbulent magnetic field (Stenflo 1971). The diagnostics of the basic building blocks of solar magnetism is challenging, because they are smaller than $100 \mathrm{~km}$ and remain unresolved even with the best telescopes of the present time.

The 2-component model was initially applied to the two Fe I lines at 5250.2 and $5233 \AA$, which brought the discovery that more than $90 \%$ of all the magnetic flux in the magnetograms of that time (which had the modest resolution of several arcsec) originated in highly clumped, strong-field magnetic elements with small filling factors in the photosphere, implying an extreme degree of intermittency (Howard \& Stenflo 1972; Frazier $\&$ Stenflo 1972). With the 5250/5233 line ratio it was however not possible to obtain quantitative estimates of the field strengths of the concentrated flux bundles, because the two lines were too different to allow the magnetic-field effects to be untangled from the thermodynamic effects. Only with the introduction of the

\footnotetext{
* Appendix is only available in electronic form at http://www. aanda.org
}

$5250 / 5247$ line ratio was it possible to isolate the magnetic-field effects (Zeeman saturation), which allowed the intrinsic field strengths to be determined to be 1-2kG (Stenflo 1973). Note that this was done in a very quiet region at disk center where the apparent field strengths (vertical fluxes divided by the area of the spatial resolution element) were of order $4 \mathrm{G}$ only.

While the introduced 2-component model was a useful mathematical tool to extract the properties of the unresolved intermittent, strong-field flux elements, it was clear from the outset that what was called the "non-magnetic" component cannot represent regions void of magnetic fields, since induction effects in the highly conductive solar plasma will generate magnetic fields everywhere. Instead it represents regions that do not contribute significantly to the net magnetic flux through the spatial resolution element. This happens if the fields are tangled on scales much smaller than the resolution element, so that there is nearly complete cancellation of the contributions from the opposite polarities. The challenge was to find a way to reveal the hidden properties of this tangled field that was invisible to the Zeeman effect. A solution to this problem came with the introduction of the Hanle effect in combination with the previously introduced concept of a micro-turbulent magnetic field, which enabled the strength of the tangled field to be constrained to lie in the range 10-100 G (Stenflo 1982).

The two complementary aspects of solar magnetism revealed by the line ratio and the Hanle depolarization effect led to a dualistic picture: intermittent kG-type flux bundles (later called 
flux tubes, although they may not always be tube like), in between which there is a tangled or turbulent field of intermediate strength. In terms of a probability density function (PDF) for the field strength such a field would be represented by two highly disjunct regions: (1) a dominating and moderately wide peak in the surroundings of zero representing the turbulent field, and a small peak (about 1\% in amplitude relative to the weakfield peak since the typical magnetic filling factor on the quiet Sun is of order $1 \%$ ) at high field strengths, of order $1 \mathrm{kG}$. This picture seemed to be at odds with numerical simulations of magnetoconvection, which all produce PDFs that connect the smallest and largest field strengths in a continuous way, without any clear indication of a dichotomy (e.g. Stein \& Nordlund 2006; Schüssler \& Vögler 2008). The observed flux densities (average of the field strength over the spatial resolution element) show the same continuous behavior as the numerical simulations, in contrast to the inferred intrinsic field strengths (as deduced from the Zeeman line ratio and the Hanle depolarization effects), which are supposed to represent the resolution-independent, resolved behavior of the field.

This apparent contradiction with the numerical simulations led to the concern that the magnetic dichotomy could be an artefact of the use of two mutually nearly exclusive diagnostic techniques. When we apply the Zeeman "diagnostic filter" we project out the kG flux tube contribution but do not see the tangled fields. When we instead apply the Hanle diagnostic filter we project out the turbulent signal while not seeing the flux tube fields.

However, we now know that this magnetic dichotomy is not an artefact of this type, since it has been revealed through applications of the Zeeman effect alone, without any reference to the Hanle effect. This was done through the application of the Stokes $V$ line ratio technique with the two $\mathrm{Fe}_{\mathrm{I}} 6301.5$ and $6302.5 \AA$ lines to a Hinode SOT/SP (Kosugi et al. 2007; Tsuneta et al. 2008; Suematsu et al. 2008) deep mode data set recorded at the center of the quiet solar disk (Stenflo 2010). The scatter plot of the Stokes $V$ amplitudes of these two lines revealed the existence of two distinct flux populations, representing strong (kG-type) and weak fields, respectively. Since two such distinct populations could be a natural product of the mechanism of convective collapse (Parker 1978; Spruit 1979; Spruit \& Zweibel 1979; Unno \& Ando 1979) we will in the present paper refer to the two populations as collapsed and uncollapsed flux, although by choosing this terminology we do not mean to rule out alternative scenarios that could lead to such a field structure. Alternatives could for instance include the dynamic pressure from the largest velocities in the solar granulation (while the convective collapse mechanism exploits the adiabatic cooling caused by downflows in the magnetic regions). Recently the convective collapse mechanism has received strong support, both from observations (Nagata et al. 2008; Fischer et al. 2009) and from realistic 3D MHD simulations (Danilovic et al. 2010).

The reason why this dichotomy had never been seen before in line-ratio data has to do with the spatial resolution. Since the opposite polarities in the weaker-field uncollapsed flux population are topologically mixed on small scales, their net contributions to the Zeeman signal averages out on scales exceeding about $1 \mathrm{Mm}$, as we will demonstrate in the present paper. Only with the Hinode resolution of $200 \mathrm{~km}$ this uncollapsed flux population begins to reveal itself. We will explore scaling laws that allow us to gain insight into the field behavior in the unresolved domain below the $100 \mathrm{~km}$ scale and to determine what is required for compatibility between the Zeeman and Hanle constraints.
The present paper represents a continuation of the detailed Hinode quiet-Sun analysis carried out in Stenflo (2010). More references to related recent work on quiet-Sun magnetism can be found in that paper.

\section{Magnetic dichotomy and the solar granulation}

We use the same Hinode SOT/SP deep mode data set of February 27, 2007, that was used by both Stenflo (2010) and Lites et al. (2008). Since on the quiet Sun the $\mathrm{S} / \mathrm{N}$ ratio is low for most of the pixels, we use a special extraction procedure (as described in great detail in Sect. 3 of Stenflo 2010) to get the most robust determination of the Stokes $V$ amplitudes. An amplitudenormalized mask has been derived from the blue lobe of the mean Stokes $V$ profile (which has a very high $\mathrm{S} / \mathrm{N}$ ratio). This mask, multiplied by a scaling factor as the single free parameter, is fit to the observed Stokes $V$ profile of each pixel. The value of the scaling parameter (with sign) represents the observed circular polarization amplitude of the blue Stokes $V$ lobe, given here in \% units of the continuum intensity. All $V$ amplitudes given in the present paper are based on the blue lobe. The similar procedure has however also been applied to the red Stokes $V$ lobe (used when the Stokes $V$ asymmetries are determined) and to the lobes of the $Q$ and $U$ profiles (for the determination of the field inclinations and azimuths).

When as in Fig. 8 of Stenflo (2010) the amplitudes of the blue Stokes $V$ lobes in the two spectral lines, $V_{6301}$ and $V_{6302}$, were plotted against each other, one could for the first time in such a scatter plot distinguish two distinct populations of points obeying two distinctly different regression relations: a dominant population following a relation with small slope as expected for intrinsically strong, $\mathrm{kG}$ type fields, and a population that is only present at smaller flux densities and that follows a larger slope that is consistent with intrinsically weak fields.

In Fig. 1 we have used brightness masking of the pixels to reveal that these two flux populations are correlated with the solar granulation. Thus the population with the smaller slope (the collapsed flux, upper panel) is preferentially located in the intergranular lanes, while the uncollapsed population (lower panel) with the larger slope prefers the interior of bright granules.

The relation between polarization and field strength is

$V \sim g B_{\|} \partial I / \partial \lambda+\ldots$,

where $g$ is the Landé factor, $B_{\|}$is the line-of-sight component of the field vector, and $\partial I / \partial \lambda$ is the gradient of the Stokes $I$ profile. If the Zeeman splitting is not small in comparison with the line width, then the higher order terms of the Taylor expansion are of importance (Zeeman saturation), but in the limit of small Zeeman splittings (here corresponding to field strengths of less than a few hundred $G$ ) only the first term is of importance. It expresses a direct proportionality between $V$ and $B_{\|}$and also shows that $V$ should be proportional to $\partial I / \partial \lambda$.

If the fields were intrinsically weak one would therefore expect that the slope in the scatter-plot diagram should equal the ratio between $g \partial I / \partial \lambda$ for the two lines. The diagonal dashed lines in Fig. 1 represent this slope, derived from the average of the blue-lobe amplitudes of $\partial I / \partial \lambda$ for each line over all pixels of the data set. This slope is thus not based on any modeling, it is based on the directly observed Stokes I profiles.

Note however that Eq. (1) becomes invalid if there are correlated velocity and magnetic field gradients along the line of sight across the line-forming layers, leading to area asymmetries of the Stokes $V$ profiles. Differential behavior between the two 
J. O. Stenflo: Collapsed and uncollapsed magnetic flux on the quiet Sun
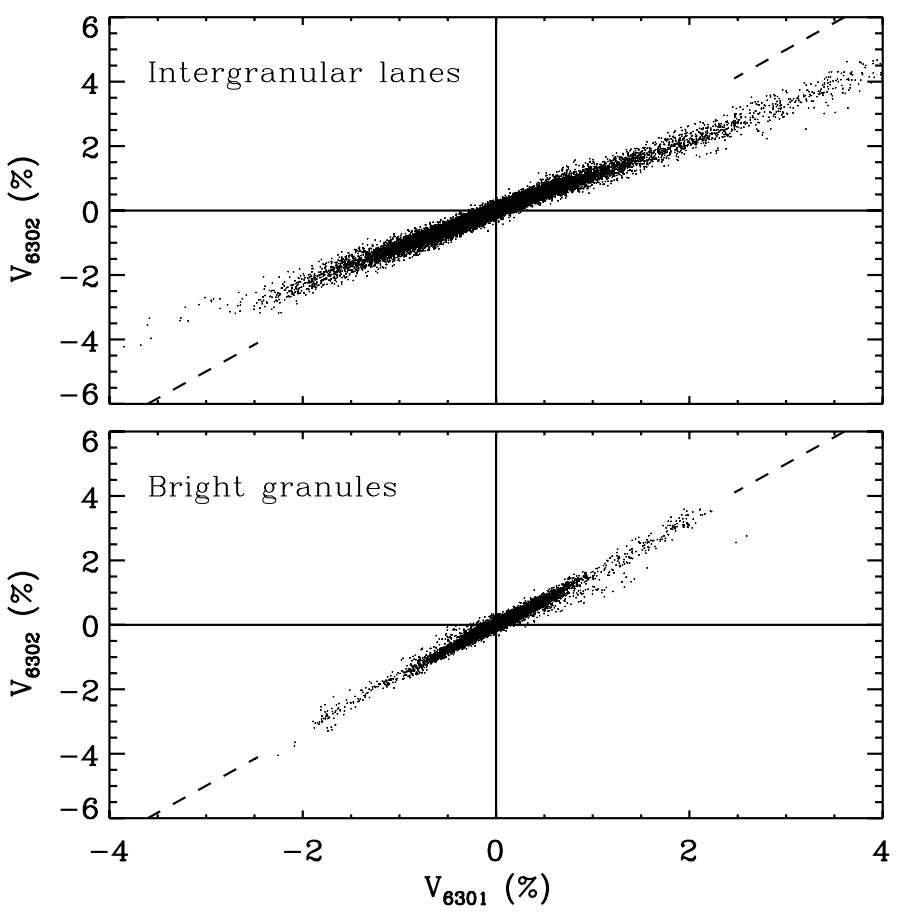

Fig. 1. Illustration of the correlation between the magnetic dichotomy and the solar granulation. Masking of the continuum brightness has been used to isolate the dark intergranular lanes and the bright cell interiors. If the fields were intrinsically weak (below a few hundred $G$ ) the points would fall along a regression line with the slope of the dashed line, as they do in the bright granules. The much smaller slope for the intergranular lanes is evidence that the magnetic flux there is in strongfield, collapsed form.

lines of this and other thermodynamic effects may produce a line ratio that in the weak-field domain systematically deviates from the ratio expected from Eq. (1) if the two lines are not formed in the same layers of the atmosphere, as is the case for the 6302 and $6301 \AA$ lines, since they have different line strengths. Although these are second-order effects, they made it necessary to renormalize the observed line ratio to that of the uncollapsed population before deriving any intrinsic field strengths for the collapsed population, as described in detail in Stenflo (2010). The differential effects are however so small that one does not notice through direct visual inspection any significant difference between the slope of the points in the lower panel of Fig. 1 and that of the dashed line.

The selection of the cuts for the brightness and velocity masking is illustrated in Fig. 2, where we have used our deepmode data set to plot the isocontours in the 2D space spanned by the relative continuum intensity (difference in $\%$ relative to the average over all pixels) and the relative Doppler velocity (difference in $\mathrm{km} \mathrm{s}^{-1}$ relative to the mean value). The intergranular lanes and the cell interiors can be isolated either by masking of the continuum intensity or of the Doppler velocity. To isolate the intergranular lanes we either select only pixels for which the continuum brightness is below a certain value or the Doppler velocity is above a certain value (redshifted). Similarly, to isolate the bright granules we choose only pixels that are either brighter or more blueshifted than a certain value.

The optimum placement of these thresholds or cuts are determined by trial and error. If we do not place the cuts sufficiently far out, then there is less clean separation between the lanes and cell interiors with resulting significant overlap between the two flux populations in the scatter-plot diagrams. If on the other hand

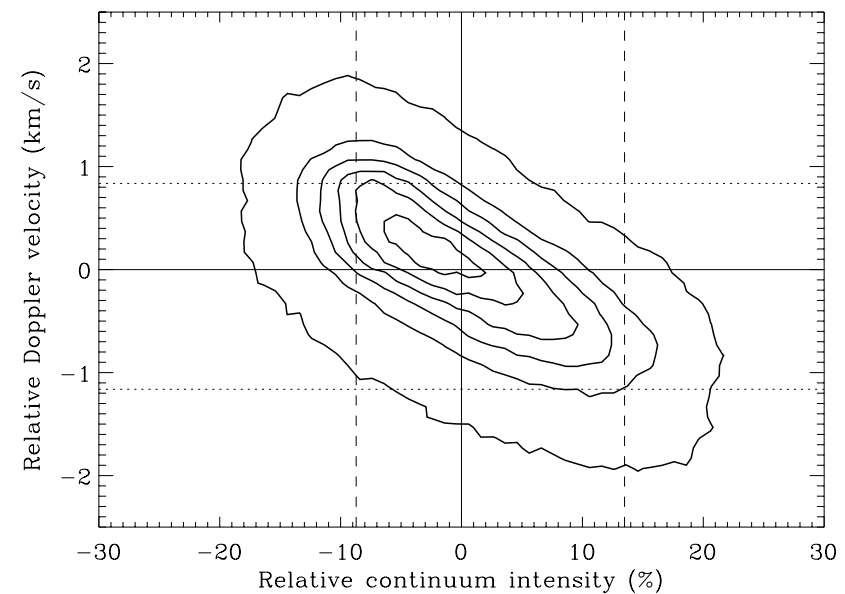

Fig. 2. Correlation between Doppler velocity and continuum brightness for solar granulation at the center of the solar disk. The vertical dashed lines mark the thresholds used for the brightness masking to isolate the intergranular lanes and cell interiors, while the horizontal dotted lines mark the corresponding thresholds used for velocity masking. The outermost contour represents the level $1 \%$ of the maximum, the innermost contour $90 \%$ of the maximum, while the in-between contours are spaced equidistantly in terms of percentage of the maximum.

we place the cuts too far out, then we are in the weak tail of the distribution with too few pixels left for good statistics. The cuts shown in Fig. 2 have been chosen by such trial-and-error optimization. The brightness cuts are marked by the vertical dashed lines, the velocity cuts by the horizontal dotted lines. For the intergranular lanes the cuts have been chosen to be at $35 \%$ of the distance from the mean value to the minimum brightness value or to the maximum redshift, for the bright granules they have been chosen to be at $45 \%$ of the distance from the mean value to the maximum brightness or to the maximum blueshift. Thus the intergranular lanes are defined as the pixels that are either darker than $-8.7 \%$ in the relative continuum intensity or more redshifted than $+0.84 \mathrm{~km} \mathrm{~s}^{-1}$, while the bright granules are defined as the pixels that are brighter than $+13.5 \%$ in the relative continuum intensity or more blueshifted than $-1.16 \mathrm{~km} \mathrm{~s}^{-1}$.

For the scatter plots in Fig. 1 we have only made use of the brightness cuts to isolate the intergranular lanes and bright granules. When one instead uses the velocity cuts the results look almost identical, so therefore they are not reproduced here.

In the 1D histograms of Fig. 3, which represent integrated versions of the $2 \mathrm{D}$ histogram of Fig. 2, we see more clearly the position of the cuts. The reason why the cuts to isolate the bright granules lie substantially further out in the tails of the distributions than the cuts to isolate the intergranular lanes is because it was necessary to go out that far to sufficiently isolate the uncollapsed population. With cuts closer to the mean value there would have been significantly more "contamination" from the collapsed population. Since the pixels representing the collapsed population are more abundant than those of the uncollapsed population, the dominance of the collapsed population can only be suppressed by isolating the outer tail of the distributions.

While we throughout the present paper only discuss and illustrate the results based on the brightness cuts, we find it worthwhile to mention that one could as well have used the velocity cuts instead, with much the same results. Our simple brightness criterion may group magnetic bright points with granules, although they are located in intergranular lanes, but this effect is statistically insignificant in the present context, in 

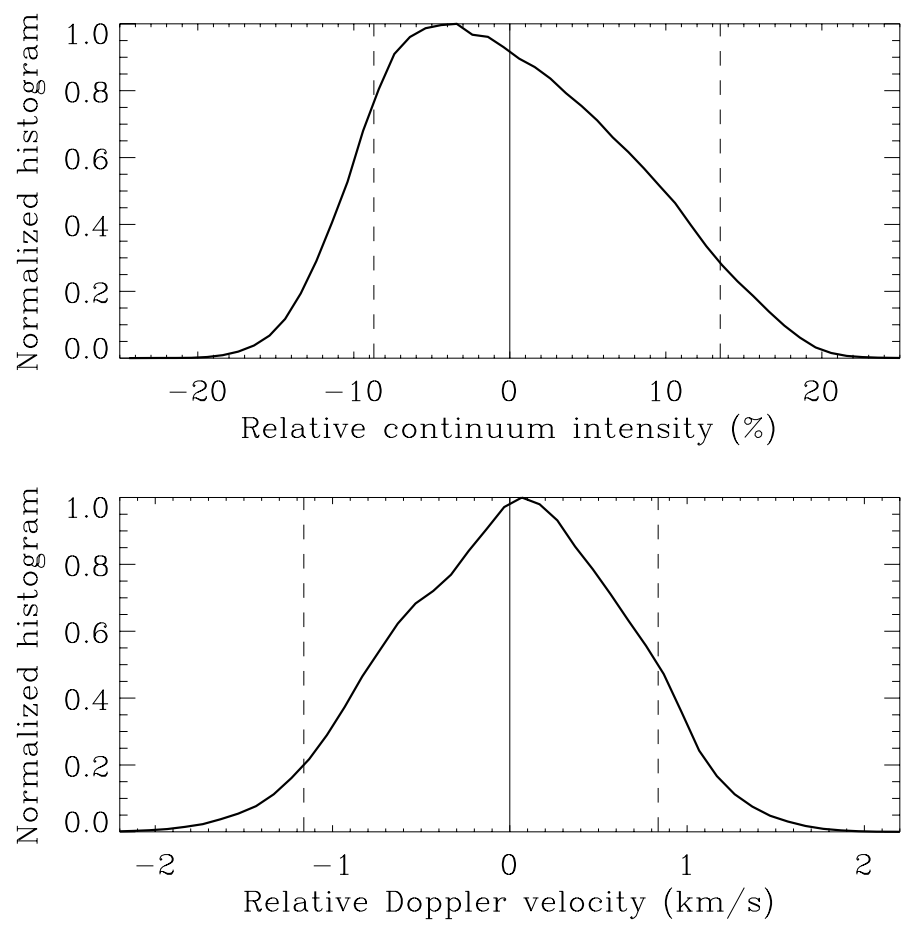

Fig. 3. Histogram of the continuum brightness (top panel) and Doppler velocity (bottom panel) derived from the 2D distribution of Fig. 2. The cuts used for the brightness and velocity masking are indicated by the vertical dashed lines, which are at the same locations as in Fig. 2.

particular since we are not using G band data but the $6300 \AA$ continuum brightness in a very quiet region.

To bring out the distinct nature of the two flux populations we show 2-component Gaussian decompositions of the line ratio in Fig. 4 for the points within a $|V|_{6301}$ bin centered at $0.95 \%$ polarization for the two cases used in Fig. 1 with brightness masking (top and middle panels of Fig. 4), and compare with the corresponding decomposition when no masking is used (bottom panel, which is the same as Fig. 9 in Stenflo 2010). In the decomposition it is assumed that the two Gaussians have the same width. The line-ratio positions of the centers of each component are marked by the vertical dotted and dashed lines. The diagonal dashed lines in Fig. 1 correspond to the relation $0.6 V_{6302} / V_{6301}=1$, which is the reason why the factor 0.6 is used in the scale for the horizontal axis. We note that the weak or uncollapsed component has in these units a position between 0.91 and 0.96 rather than 1.0 , which most likely is not caused by any Zeeman saturation (intrinsically strong fields) but by differential thermodynamic effects in the two unequally strong lines.

Comparing with the bottom panel we see how astonishingly well the brightness masking has succeeded in bringing out each flux component alone. The intergranular lanes are almost exclusively occupied by collapsed flux with very little "contamination" from the uncollapsed component. In contrast, it is not possible to completely avoid mixing of the two components by masking of the bright granules, there is always substantial overlap from the collapsed component, although the inside of the granules is clearly the preferred location of the uncollapsed flux.
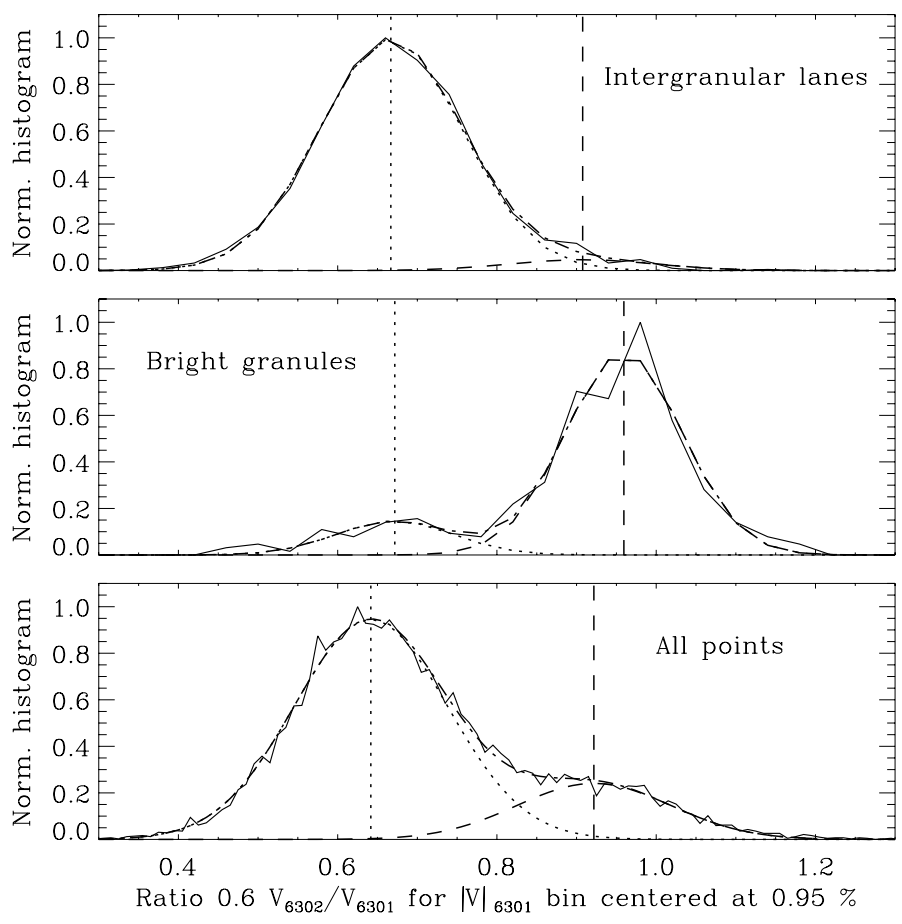

Fig. 4. Histogram distributions (solid curves) for the Stokes $V$ line ratio along a vertical cut through the scatter-plot diagrams within a $|V|_{6301}$ bin centered at $0.95 \%$ polarization. The dashed-dotted curves represent 2-component Gaussian decompositions, with each Gaussian given by the dotted and dashed curves. The center positions of the two Gaussians are marked by the vertical dotted and dashed lines. The top and middle panels are derived from the top and bottom scatter plots of Fig. 1, while the bottom panel represents the case with no masking.

\section{Stokes $V$ line ratio and thermodynamics}

\subsection{Masking of the line ratio}

The rather clean separation between the collapsed and uncollapsed components that we achieve by our brightness masking in Figs. 1 and 4 may give the impression that there is little overlap between the two components at a given position on the Sun. This impression may however be a bit misleading, since we had to exploit the outer tails of the brightness distributions to avoid substantial overlap. To get a more truthful assessment of the situation we have therefore reversed the masking procedure: Instead of doing the brightness masking of Fig. 2, we do masking of the Stokes $V$ line ratio to determine where the pixels with small and high values of the line ratio fall in brightness-velocity space, with the result illustrated in Fig. 5.

The cuts used for the masking of the line-ratio values to isolate each flux population have to be set so far apart that there can be no significant contamination through partial overlap of the distributions. Thus the partial overlap region between the two Gaussians that we see in Fig. 4 should be avoided. As we go to smaller polarization amplitudes (or flux densities) the overlap increases, because the closer we come to the origin of the coordinate system in the scatter plots of Fig. 1, the more difficult it becomes to distinguish between regressions with different slopes.

To isolate the collapsed flux population we have made the cuts such that only pixels for which $0.6 V_{6302} / V_{6301}$ (the quantity used for the horizontal axis in Fig. 4) falls between 0.4 and 0.7 , and for which the $1-\sigma$ error in this quantity (as derived from the measured 1- $\sigma$ errors in the Stokes $V$ polarization values) is 
J. O. Stenflo: Collapsed and uncollapsed magnetic flux on the quiet Sun
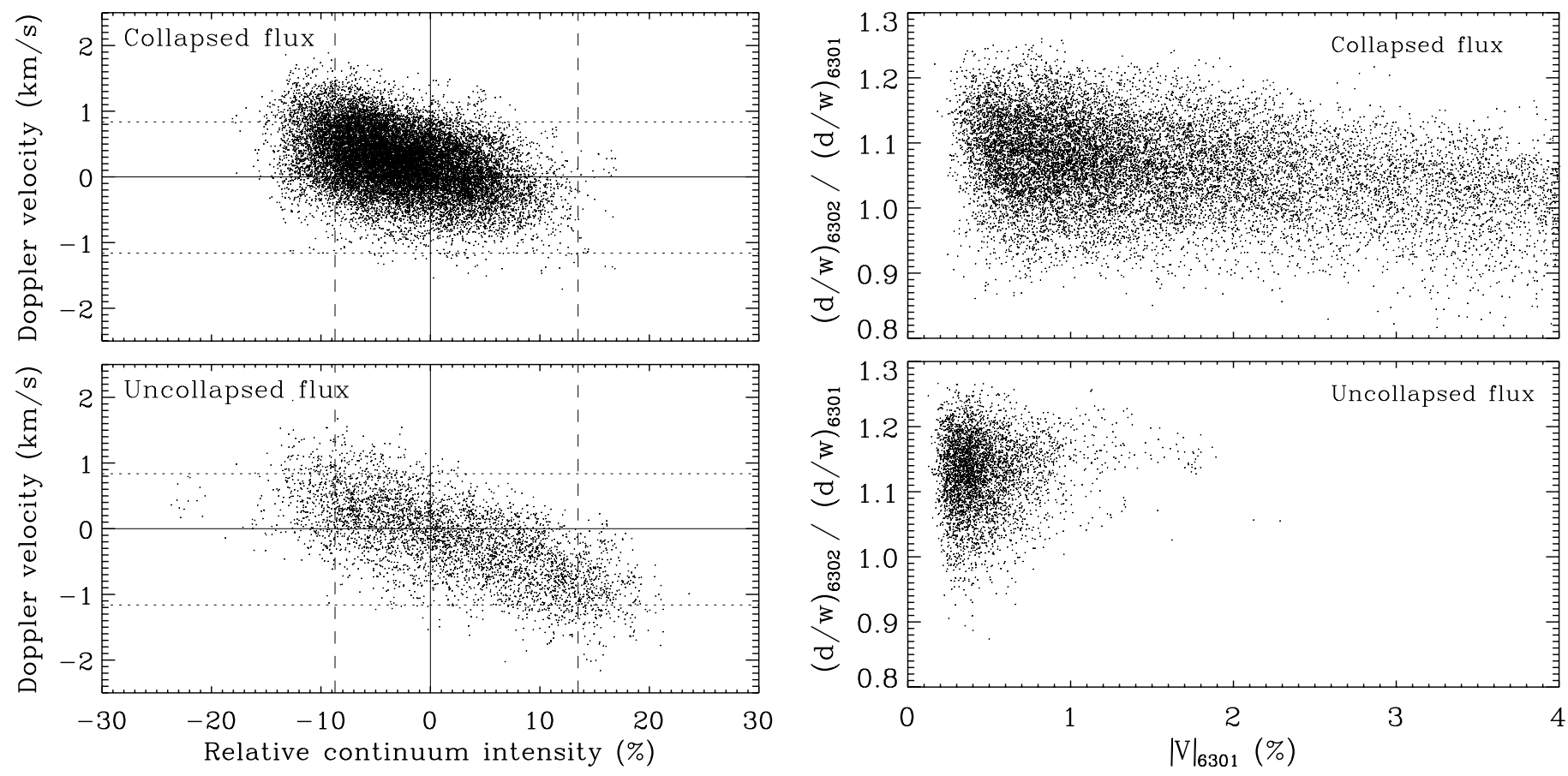

Fig. 5. Distributions of the pixels in brightness-velocity space when masking of the Stokes $V$ line ratio is used. Top panel: only pixels with small line ratio, representing the collapsed flux population. Bottom panel: only pixels with large line ratio, representing the uncollapsed population.

smaller than 0.07 . The corresponding cuts to isolate the uncollapsed flux population are defined by selecting only the pixels for which the above ratio falls within the interval between 0.9 and 1.2 , and for which the $1-\sigma$ error is smaller than 0.15 . This compromise choice should give a sufficient separation of the populations. The reason why we have chosen to be more restrictive with the 1- $\sigma$ error for the collapsed as compared with the uncollapsed population is that the dominating collapsed population with its abundance of pixels allows this while still offering a large statistical sample. In contrast, the great majority of the pixels that represent the uncollapsed flux population have small flux densities, and the standard error in the line ratio increases rather steeply with decreasing flux density. If we choose a more restrictive 1$\sigma$ criterion for the uncollapsed flux, then our statistical sample becomes marginally small.

Figure 5 shows that the two flux populations are not at all as well separated in brightness-velocity space (i.e., not as well correlated with the solar granulation) as Fig. 1 could lead you to believe. Instead the main message of Fig. 5 is that it is the spatial and morphological overlap between the two flux populations that is the dominating property. To second order, however, the two distributions in brightness-velocity space differ. Thus the distribution that represents the collapsed flux is systematically shifted towards the upper left, i.e., towards the darker and redshifted regions, and away from the brighter and blueshifted regions. This has the effect that there are very few points left that lie to the right of the cut that selects the bright regions (brightness larger than $13.5 \%$ ), while at the same time a significant fraction of the pixels that represent the uncollapsed flux falls in this domain. This explains why the scatter plot in the lower panel of Fig. 1 for the bright granules is dominated by points following the slope that represents the intrinsically weak fields. At the same time there is a great number of points to the left of the dark cut (brightness less than $-8.7 \%$ ), with an abundance that overwhelms the

Fig. 6. Here a line ratio is formed for the ratio between line depth $d$ and line width $w$ : $(d / w)_{6302} /(d / w)_{6301}$. It is plotted vs. the polarization amplitude $|V|_{6301}$ in combination with Stokes $V$ ratio masking. The same masking criteria have been used as in Fig. 5. Thus the top panel represents pixels with small $V$ ratio (collapsed flux population), the bottom panel pixels with large $V$ ratio (uncollapsed flux population).

much smaller contribution from the uncollapsed flux that mainly populates the region in the scatter plot closer to the origin of the coordinate system with small polarization amplitudes. This has the consequence that it is only the collapsed population with the small regression-line slope that has good visibility in the scatter plot for the intergranular lanes (top panel of Fig. 1).

In contrast to the collapsed population, the distribution in brightness-velocity space of the uncollapsed population (lower panel of Fig. 5) remains rather similar to the overall distribution of Fig. 2, with only a slight shift, if any, towards brighter and more blueshifted regions. This shows that while the collapsed flux population is significantly correlated with the intergranular lanes, the uncollapsed flux population has a very weak correlation with the solar granulation with little difference in preference between the bright granules and the intergranular lanes.

\subsection{Role of $\partial I / \partial \lambda$}

Since there is a statistical correlation between the Stokes $V$ line ratio and the solar granulation, and since there is a significant difference in thermodynamic environment and line formation behavior between the bright granules and the intergranular lanes, the justified question arises if the observed dichotomy in the line ratio, or at least part of it, could be an artefact of this environmental difference rather than being caused by Zeeman saturation due to intrinsically strong fields. The main way in which the thermodynamics affects this issue is through its effect on the scaling relation between $V$ polarization amplitude and vertical flux density $B_{\|}$(which at disk center is along the line of sight). The relation is given by Eq. (1), with the scaling factor in the weak field regime (when only the first term in the Taylor expansion is needed) being determined by the Stokes $I$ gradient $\partial I / \partial \lambda$. 
For a given line profile shape $\partial I / \partial \lambda$ scales with $d / w$, the ratio between relative line depth $d=\left(I_{\mathrm{c}}-I\right) / I_{\mathrm{c}}$ and line width $w$ (at the level of half the line depth). If the Stokes $V$ line-ratio variations were due to thermodynamic environment effects rather than to varying Zeeman saturation, then the $(d / w)_{6302} /(d / w)_{6301}$ ratio should behave like the Stokes $V$ ratio, and one should find a dichotomy in the $d / w$ line ratio. We have therefore applied the same $V$ ratio masking as was used for Fig. 5 to plot in Fig. 6 the $(d / w)_{6302} /(d / w)_{6301}$ line ratio as a function of the $|V|_{6301}$ polarization amplitude. Because the relative abundance of the uncollapsed flux population decreases steeply with increasing flux density (or polarization amplitude $|V|_{6301}$ ), there is a scarcity of points beyond a polarization of $1 \%$, while the collapsed population extends to large flux densities. Secondly, due to the 1- $\sigma$ error limits used together with our $V$ ratio cuts, the inner region with small polarization amplitudes is empty, because there the standard error in the $V$ ratio exceeds our chosen limits, leading to the exclusion of these pixels.

We see in the panel for the collapsed flux that there is a slope in the scatter-plot distribution: the $(d / w)_{6302} /(d / w)_{6301}$ ratio decreases as $|V|_{6301}$ increases. This can be understood as a natural consequence of the circumstance that the flux tubes of the collapsed component have very different internal thermodynamic properties (with very strong line weakenings) than the surrounding atmospheric environment, regardless of whether the flux tubes are sitting in the intergranular lanes or in bright cell interiors. Therefore the $(d / w)_{6302} /(d / w)_{6301}$ ratio of the insides of the flux tubes is significantly different from that of the surroundings. With increasing polarization amplitude the filling factor of the collapsed component increases in a way that is almost linear to a first approximation. Since the proportion of flux tube atmosphere within the resolution element scales with the filling factor we expect the $(d / w)_{6302} /(d / w)_{6301}$ ratio to vary in an almost linear way with polarization amplitude, as observed in Fig. 6. The large influence of the internal thermodynamics through the line weakenings was carefully accounted for in the conversion of the Stokes vector data to flux densities and inclinations in Stenflo (2010), as described in great detail in that paper.

The issue here is however not how the internal thermodynamics affects the interpretation, but to what extent the observed dichotomy in the Stokes $V$ line ratio could be an artefact of distinctly different thermodynamic environments between the intergranular lanes and the bright cell interiors. To determine such effects we should compare the $(d / w)_{6302} /(d / w)_{6301}$ line ratios of the two flux populations in the limit of small flux densities (small polarization amplitudes). If we take the average of $(d / w)_{6302} /(d / w)_{6301}$ for the points in Fig. 6 with polarization amplitudes below $1 \%$, then we find an average ratio of 1.09 for the collapsed flux population, 1.13 for the uncollapsed, thus a difference of about $4 \%$. Due to the slope of the points for the collapsed population this difference becomes smaller in the limit of vanishing filling factor.

In contrast the Stokes $V$ line ratio of the collapsed population is typically only about $70 \%$ of that of the uncollapsed population. The relative difference between the two $V$ ratio populations is therefore an order of magnitude larger than what we find for $(d / w)_{6302} /(d / w)_{6301}$. This demonstrates that the magnetic dichotomy exposed by the $V$ line ratio cannot be an artefact of the varying thermodynamics in the solar granulation.

If we had been able to use an ideal magnetic line ratio with two lines of the same strength that are formed in the same way in the solar atmosphere apart from having different Landé factors, like the 5250/5247 line ratio (Stenflo 1973), then we would have expected the ratio $(d / w)_{5250} /(d / w)_{5247}$ to be unity everywhere. It would be interesting to verify this in future observations with the $5250 / 5247$ line ratio. It is gratifying that our $(d / w)_{6302} /(d / w)_{6301}$ ratio differs from unity on average by only about $10 \%$, which verifies that this line ratio is still quite useful for magnetic-field diagnostics in spite of the different line-formation properties of the two lines.

\subsection{Stokes $V$ asymmetry and solar granulation}

While the magnetic dichotomy with two flux populations thus is not an artefact of the different environments of intergranular lanes and cell interiors, there are other interesting and intriguing ways in which the thermodynamics can affect the Stokes profiles. One such property of potentially great diagnostic value is the Stokes $V$ line asymmetry $A$, defined by

$A=\frac{V_{\text {blue }}+V_{\text {red }}}{V_{\text {blue }}-V_{\text {red }}}$

Here $V_{\text {blue }}$ and $V_{\text {red }}$ are the amplitudes, with sign, of the blue and red lobes of the Stokes $V$ profile. Then $A$ represents the $a m$ plitude asymmetry. If we instead would have used the areas of the corresponding $V$ lobes, then $A$ would have represented the area asymmetry (which we will not use here, since it is more affected by noise, and on the quiet Sun we are dealing with very weak signals). In static atmospheres the $V$ profiles are perfectly anti-symmetric, with the result that $A$ is always zero. $A$ can differ from zero only if there are correlations between the gradients of the magnetic and velocity fields on spatially unresolved scales within the resolution element. The area asymmetry can differ from zero only if these correlations are along the line of sight, while the amplitude asymmetry can also be due to correlated gradients perpendicular to the line of sight (cf. Stenflo et al. 1984).

The histogram analysis of the Stokes $V$ amplitude asymmetries for our Hinode SP deep mode data set done in Stenflo (2010) showed that for small flux densities the distribution of asymmetry values is extremely wide, with high probability for all kinds of profiles. Thus either the blue or the red lobe can be the dominating one, and there are many cases where both the blue and red lobes are of the same sign (as happens when $A>1$ ). As the flux density increases the distribution becomes narrower. For high flux densities all profiles tend to converge to a common shape. However, the histogram distributions are systematically shifted to the positive side of zero asymmetry for all flux densities, meaning that on average the blue $V$ lobe dominates over the red lobe.

To see how the Stokes $V$ asymmetry differs between the intergranular lanes and the cell interiors we have applied the same brightness masking as was used for Fig. 1 and derived histograms for the amplitude asymmetry for different bins in the vertical flux density. Figure 7 shows the results for the bins centered around the flux density values $7,17,54$, and $130 \mathrm{G}$. The solid curves represent the intergranular lanes, the dashed curves the bright cell interiors. Since this brightness masking was found to separate the two flux populations so well, we can also consider the solid curves to represent the collapsed flux population, the dashed curves the uncollapsed population.

We notice in Fig. 7 the previously mentioned property that for small flux densities the distribution is extremely wide, with an abundance of pixels with $A<0$ (representing red-lobe dominated profiles) and a large tail extending into the domain $A>1$ (representing lobes of the same sign). The distributions get narrower with increasing flux density. The most striking feature of 
J. O. Stenflo: Collapsed and uncollapsed magnetic flux on the quiet Sun
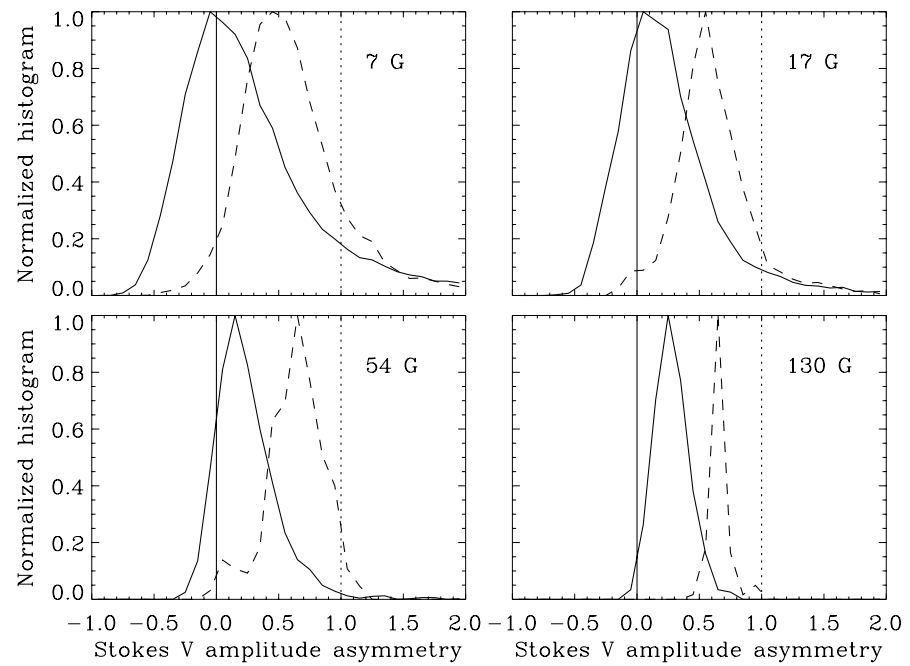

Fig. 7. Normalized histograms of the Stokes $V$ amplitude asymmetries for different bins in the vertical flux density, centered around 7, 17, 54, and $130 \mathrm{G}$. The same brightness masking as used for Fig. 1 has been applied. Thus the solid curves represent the darkest areas (intergranular lanes), the dashed curves the brightest areas (cell interiors). The vertical solid line marks zero asymmetry, to the left of which the red $V$ lobe dominates, while the blue lobe dominates to the right. The dotted vertical line marks an asymmetry of unity. To the right of this line both lobes have the same sign.

Fig. 7 is however the large systematic difference between the cell interiors and the intergranular lanes: the $V$ asymmetry is on average much larger in the cell interiors than in the intergranular lanes. This is surprising, since one might have expected the opposite, namely that the effect of spatial gradients would be larger in the lanes than in the cell interiors. It is a result that is not easy to interpret, and one would need detailed 3D modeling to identify the causes. In any case this surprising property may serve as a powerful constraint on the magnetohydrodynamic structure of the solar granulation.

The plot in Fig. 7 represents the $6301 \AA$ line. We have made the same histogram plots also for the $6302 \AA$ line, but as the behavior is the same, we do not reproduce these plots here. We thus see no evidence for differential behavior of the Stokes $V$ asymmetries between the two lines.

Note that the rather clean separation between the histograms for the lanes and the cell interiors has been achieved through a choice of brightness cuts that isolates the extreme tails of the brightness distributions, in the same way as this allowed us to get a rather clean separation between the two flux populations. For the great majority of pixels there is a substantial mixture of the two histogram distributions, regardless of whether the pixel is located in a lane or in a cell interior, as we saw in Fig. 5 for the $V$ ratio.

\section{Scaling behavior of the vertical flux densities}

\subsection{Scaling of the scatter plot}

The reason why the magnetic dichotomy has never been seen in earlier line-ratio data is because one needs the high spatial resolution of Hinode to see it. Therefore we will next use our Hinode deep mode data set to simulate how the Stokes $V$ scatterplot results depend on the spatial resolution of the instrument, through box-car smoothing of the $V$ arrays.
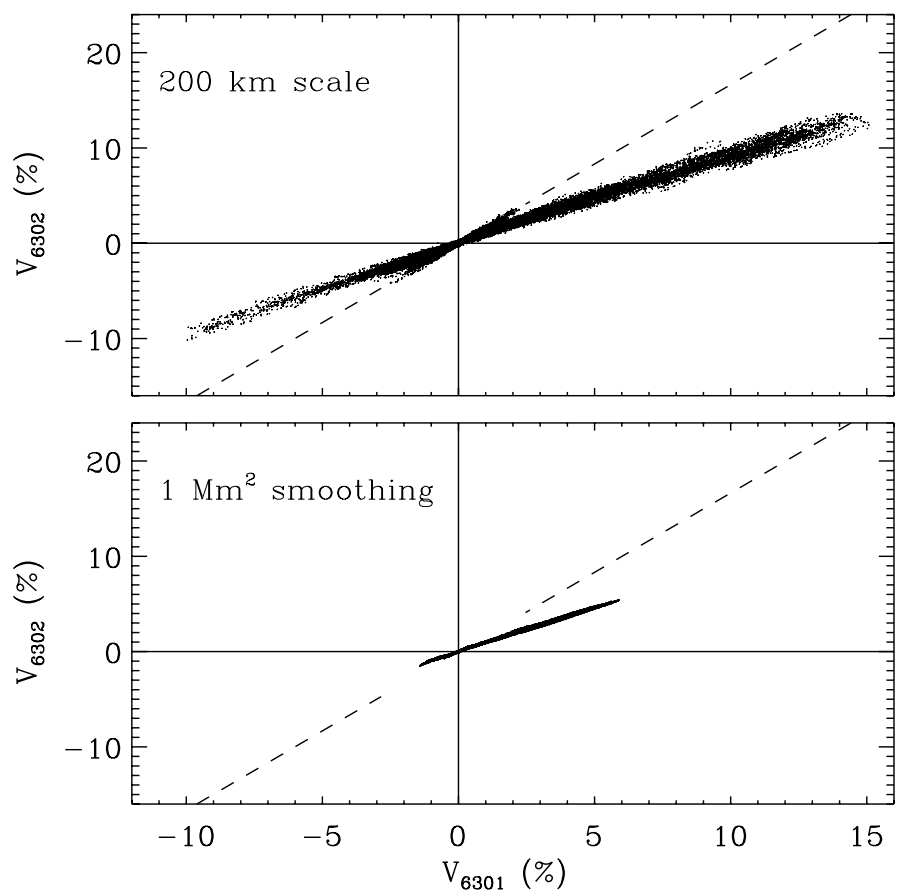

Fig. 8. Scatter plots of $V_{6302}$ vs. $V_{6301}$ for the Hinode deep mode data set. The top panel shows the plot for all pixels at the original Hinode resolution (same as Fig. 8 of Stenflo 2010), while the bottom panel shows what happens after the same data set has been spatially smoothed with a window of area $1 \mathrm{Mm}^{2}$. If the magnetic fields were intrinsically weak (less than a few hundred $\mathrm{G}$ ), the points would fall along the dashed line.

Since the deep mode recordings use a slit at a fixed diskcenter position without spatial scanning (only tracking of the solar rotation) to optimize the $\mathrm{S} / \mathrm{N}$ ratio, we can only do spatial smoothing in one dimension, along the slit. This can be converted into an equivalent area smoothing if we for the resolution element in the direction perpendicular to the slit assume twice the usual pixel size of 0.15 arcsec, which is about $215 \mathrm{~km}$. If we for instance smooth with a box that is $4.7 \mathrm{Mm}$ along the slit, the area over which we have smoothed will be $4.7 \times 0.215=1 \mathrm{Mm}^{2}$. If we describe this area as $d^{2}$, then the introduced scale size $d$ represents the side of a square box that has the same area as used for smoothing with a differently shaped box.

We will for convenience often refer to the term "line-ratio scatter plot" although what we mean refers to plots of the type in Fig. 1 with the $V$ amplitude of each line plotted against each other, and not to the $V$ ratio itself, which is instead represented in the form of the slopes of the regression relations. In Fig. 8 we show how the line-ratio scatter plot changes as we go from the original Hinode resolution (of about $200 \mathrm{~km}$, top panel) to the described smoothing of $1 \mathrm{Mm}^{2}$ (bottom panel). Again, the dashed line marks the slope along which the points would fall if the contributing fields were intrinsically weak. In the inner part of the top panel (same as Fig. 8 of Stenflo 2010) we notice the presence of the secondary population of points that follow the slope of the dashed line and therefore represents the uncollapsed fields. However, in the bottom panel it has vanished, there is no trace of it. The reason is that the uncollapsed flux population consists of flux elements with polarities that are mixed on scales smaller than the $1 \mathrm{Mm}$ scale. Therefore it is only the collapsed flux population that survives such polarity cancellation and contributes on scales larger than $1 \mathrm{Mm}$. The transition occurs at substantially smaller scales. Already with $0.5 \mathrm{Mm}$ smoothing it is hard to see any remaining trace of the uncollapsed population. 
One really needs the Hinode resolution to see it. However, even with the Hinode resolution, this population only gets marginally revealed. We may expect that it will become increasingly more visible as the angular resolution of our instruments improves. The scaling law for the visibility of the mixed-polarity flux component will be derived and shown in Fig. 10 below.

This result confirms the early conclusion from the first applications of the Stokes $V$ line ratio that more than $90 \%$ of the total flux seen in magnetograms with the resolution of that time originates in strong-field $(\mathrm{kG})$ flux elements in the photosphere. Thus Howard \& Stenflo (1972) concluded that the line-ratio data showed that more than $90 \%$ of the total magnetic flux seen with the $17 \times 17 \operatorname{arcsec}^{2}$ resolution of the Mt Wilson magnetograms was in strong-field form, while Frazier \& Stenflo (1972) found that more than $90 \%$ of the total magnetic flux seen with the $2.4 \times 2.4 \operatorname{arcsec}^{2}$ resolution of the Kitt Peak magnetograms was in strong-field form. It was totally clear and obvious already then that the $90 \%$ flux statement was a resolution-dependent statement and referred to the net, uncancelled flux within the spatial resolution element that contributes to the signal in the magnetograms. It was clear that the observations provided no information on the amount of cancelled, mixed-polarity flux within the resolution element, since this flux is hidden, invisible to the Zeeman-effect observations. It was only with the introduction of the Hanle effect that the existence and magnitude of this hidden field could be revealed (Stenflo 1982). It was also clear that as the angular resolution of our instruments increases, we would begin to partially resolve this mixed-polarity field and make it partially visible in the Zeeman-effect observations. We now see that this begins to happen as we reach the Hinode $200 \mathrm{~km}$ resolution.

When comparing the top and bottom panels of Fig. 8 we notice two other effects: (1) the scatter around the regression line has been greatly reduced by the averaging of the random fluctuations (in particular the spread due to measurement noise). (2) the range of polarization amplitudes covered by the data set has shrunk dramatically. To clarify this second effect we show in Fig. 9 how the maximum polarization varies with scale size $d$ (as defined above) of the smoothing window. The vertical thin solid line marks the Hinode scale, from which we start the scaling. The upper solid curve represents the maximum polarization at the right end of the scatter plot, after conversion of polarization amplitude to vertical flux density in $\mathrm{G}$ (as described in Stenflo 2010). The lower solid curve shows the largest negative polarization at the left end of the scatter plot, also after conversion to G. The two dashed curves represent $1 / d^{2}$ fits to the tails of the solid curves.

The way in which one can understand this scaling behavior is as follows: the great majority of the pixels have small flux densities near the origin of the coordinate system in the scatter plots. The pixels with the largest flux densities within the field of view are scarce and most likely represent isolated "hills" surrounded by pixels with small flux densities and mixed polarities. These "hills" have a certain spatial size (although they are spatially unresolved in the sense that the filling factor is smaller than unity). When the size of our smoothing window is smaller than or comparable to the size of the flux clump, then the apparent flux density declines only slowly with increasing scale size $d$. However, in the regime where the smoothing window is significantly larger than the size of the clump we only dilute the polarization contribution from the flux concentration by adding more of the negligible net contribution from the weak surroundings. As the relative area occupied by the flux concentration within the smoothing window is inversely proportional to the area of

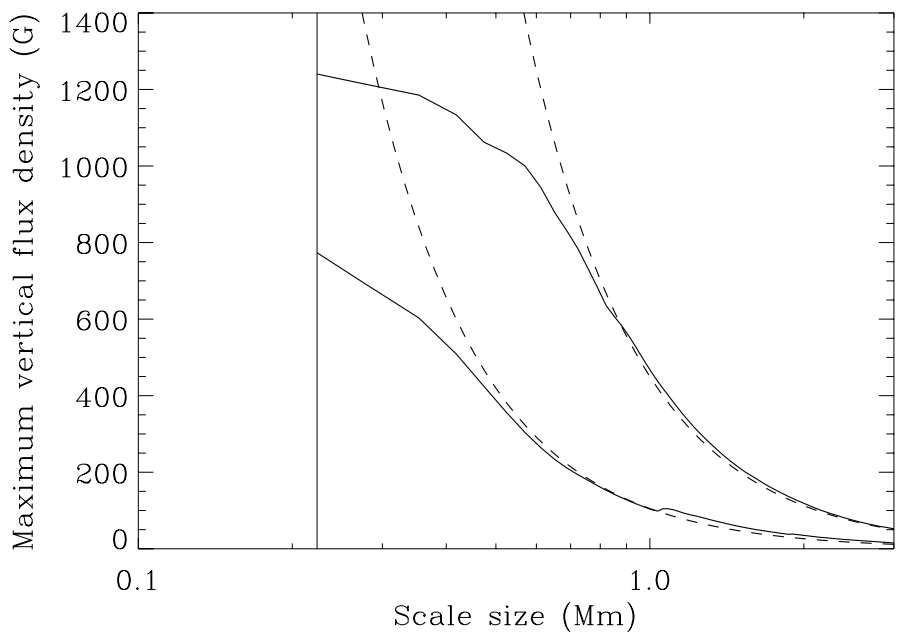

Fig. 9. Illustration of the scaling behavior of the largest positive (upper solid curve) and negative (lower solid curve) flux density, representing the extreme right and left points of the Stokes $V$ scatter plots after conversion to $\mathrm{G}$ units. $d$ is the scale size of the smoothing window, as defined in the text. The dashed curves represent $1 / d^{2}$ fits to the tails of the solid curves, demonstrating that the extreme $V$ polarizations represent isolated flux density concentrations with sizes below $1 \mathrm{Mm}$. The thin vertical solid line marks the Hinode resolution scale.

the smoothing window, we expect the flux density to decrease as $1 / N$, where $N$ is the number of pixels within the smoothing window, i.e., also as $1 / d^{2}$, since $d^{2}$ per definition is the area of the smoothing window. The transition to the $1 / d^{2}$ behavior occurs already at scales below $1 \mathrm{Mm}$. We have inspected the spatial profiles along the slit of the maximum "hills" to verify that they are consistent with the scaling behavior in Fig. 9.

Let us clarify a small peculiarity in Fig. 9. Close inspection reveals a slight increase of the lower solid curve near a scale of $1 \mathrm{Mm}$, while the above discussion may lead to the expectation of a strictly monotonic behavior of the curves. However, this discussion referred to isolated flux "hills" that get smoothed out. When the smoothing window gets so wide that it begins to cover more than one major flux clump, then the average flux within the window can increase in spite of the larger averaging area.

\subsection{Cancellation function}

Let us now turn to a different scaling law called cancellation function, introduced by Pietarila Graham et al. (2009). It describes how the average (over all the pixels) of the unsigned vertical flux density $B_{\mathrm{v}}$ changes with scale size (of the $2 \mathrm{D}$ angular resolution element). Over the range of scales for which the magnetic field has a self-similar scaling behavior the cancellation function should behave like a power law:

$\left\langle\left|B_{\mathrm{v}}\right|\right\rangle \sim d^{-\chi}$,

where $\chi$ is the cancellation exponent. Analysing a Hinode SOT/SP data set at the quiet-Sun disk center Pietarila Graham et al. (2009) found a cancellation exponent $\chi \approx 0.26$ and concluded that at least $80 \%$ of the total vertical magnetic flux remains invisible to Hinode due to cancellation of the mixed polarities inside the Hinode $200 \mathrm{~km}$ resolution element.

If the whole field of view were unipolar, then the average of the unsigned flux density would not depend on the degree of spatial smoothing, and the cancellation exponent would be zero. If, in the other extreme, the flux density in each pixel would be mutually uncorrelated and randomly fluctuate around zero, as they 
would for random noise, then the average of the unsigned flux density would vary like $1 / \sqrt{N}$, where $N$ is the number of pixels within the smoothing window. Since $N \sim d^{2}$, the cancellation exponent would be unity. The value 0.26 represents an intermediate behavior, as expected for a fractal pattern.

For the empirical derivation of the cancellation function the deep mode SP data are not suited, since they only allow smoothing in one spatial dimension (along the slit). Although it is possible even in this case to define an area for the smoothing window and let the square root of this area define the scale size $d$ of the smoothing, as was done for the scatter plots, this procedure is not equivalent to smoothing with a square-shaped window. The chosen shape of the smoothing window is not critical for the result, but the aspect ratio between the sides of the smoothing box should remain constant as the scale size is changed. This is not the case when we smooth along the slit only, since the smoothing window gets more elongated as the smoothing is increased. For this reason Pietarila Graham et al. (2009) instead used an SP data set with step-wise spatial scanning that builds up a 2D field of view, since this allowed them to use a square-shaped smoothing window. A potential problem with this type of Hinode data set is that due to the slow scanning the spatial and temporal variations get mixed in the scanning direction. Across a distance of a few arcsec the magnetic field gets statistically uncorrelated due to field evolution. For a smoothing range less than a few arcsec, however, these effects should be minor.

To verify the results of Pietarila Graham et al. (2009) we have analysed with a square-shaped smoothing window the same 2D Hinode SOT/SP data set as used by them, and we indeed find approximately the same cancellation exponent if we either use for the vertical flux densities the values obtained with the Lites et al. (2008) reduction, or if we simply derive it directly from the Stokes $V$ map. This is to be expected since the flux densities of Lites et al. (2008) are almost directly proportional to $V$ (with minor saturation for large $V$ values). In contrast, when using the reduction pipeline of Stenflo (2010) to get from $V$ to vertical flux densities, we find a cancellation exponent that is only about half as large. This is surprising, because when the vertical flux density values of Lites et al. (2008) and Stenflo (2010) are plotted against each other they show an excellent correlation. This result indicates that the derived value of the cancellation exponent is very sensitive to the way in which the flux densities are determined from the observed polarizations.

The cause for this surprising discrepancy seems to lie in the relative weights that we implicitly give to the collapsed and uncollapsed flux populations. In the analysis procedure of Stenflo (2010) great care is taken to account and correct not only for the Zeeman saturation of the intrinsically strong fields, but in particular for the large intrinsic line weakenings within the collapsed flux tubes. These corrections lead to a scaling up of the apparent flux densities of the collapsed population by a factor of order two, thus giving substantially more weight to the extended tails of the PDF. As will be seen later in Fig. 14, the magnitudes of the unsigned average flux densities are largely determined by the tail of the PDF, which is composed of the stronger and more isolated flux concentrations and therefore does not contribute much to the cancellations of the opposite polarities. Most of this cancellation is expected to be due to the inner part of the PDF with the small flux densities, where in particular the uncollapsed flux population resides. With our corrections for the intrinsic properties of the collapsed population we implicitly give significantly larger weight to the PDF tails as compared with the Lites et al. (2008) reduction that does not make use of any corrections of this kind.

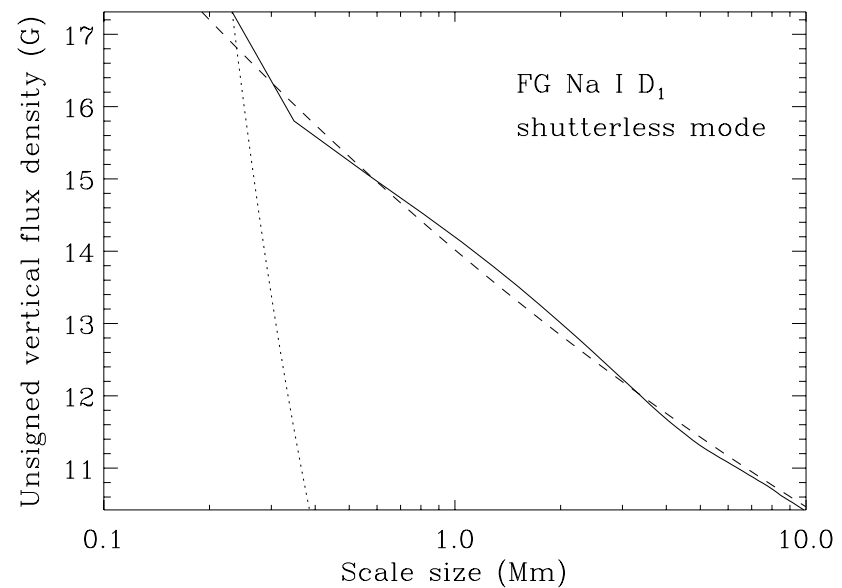

Fig. 10. Cancellation function, defined as the average of the unsigned vertical flux density, as a function of scale size $d$ of a square-shaped smoothing window. It has been derived from a shutterless Hinode SOT/FG data set obtained on November 14, 2009 (solid line). The dashed line is a fit with the function $d^{-0.127}$, while the dotted line describes the behavior of random noise.

To avoid such issues we have here chosen to derive the cancellation function from a 2-hour Hinode SOT/FG data set obtained on November 14, 2009, in shutterless mode (which provides a $\mathrm{S} / \mathrm{N}$ ratio that is better by a factor of 3.5 as compared with the usually used shuttered mode). This data set contains several hundred filter magnetograms recorded in the wing of the $\mathrm{Na}$ I $\mathrm{D}_{1}$ line, which is a broad line formed in the lower chromosphere. The magnetograms represent Stokes $V$ maps with an uncalibrated polarization scale, which we have converted to flux densities in $\mathrm{G}$ through regression analysis with vertical flux densities derived (using the reduction pipeline of Stenflo 2010) from an overlapping narrow field of view of simultaneous SP observations in the 6301 and $6302 \AA$ lines. This calibration however only provides a global conversion factor, so for the purpose of determining the cancellation exponent, it is entirely immaterial whether or not we use the converted or unconverted $V$ map.

The use of the Na I $\mathrm{D}_{1}$ Stokes $V$ maps rather than SP maps has two main advantages: (1) A 2D map is obtained simultaneously over the whole field of view. As there is no scanning, there is no mixing of the spatial and temporal fluctuations. (2) Since the $\mathrm{Na}_{\mathrm{I}} \mathrm{D}_{1}$ line is a broad chromospheric line that is not significantly affected by intrinsic line weakenings and Zeeman saturation, the assumption of direct proportionality between Stokes $V$ and vertical flux density should be valid, in contrast to photospheric lines like the 6301 and $6302 \AA$ lines. Therefore we avoid issues relating to uncertainties in the conversion from polarization to flux density, since we can use the $V$ map directly.

Since the FG magnetograms are based on the use of a filter pass band at a fixed position in the line wing, there may be a concern that Doppler shifts, which are spatially correlated with the solar granulation, may contaminate the data. This contamination can however only happen due to variations of the Stokes $V$ scaling factor $\partial I / \partial \lambda$, when one samples different portions of the line wing. As the pass band is placed near the inflexion point of the line wing, such scale variations are nearly zero to first order, when the Doppler shift is small compared to the line width. Since the distance between the inflexion point and the line center is approximately $9 \mathrm{~km} \mathrm{~s}^{-1}$ in velocity units for the broad $\mathrm{Na}$ I $\mathrm{D}_{1}$ line, much larger than the Doppler shifts of the solar granulation, Doppler contamination of the magnetograms is insignificant. 
The resulting cancellation function as derived from this SOT/FG data set is shown as the solid line in Fig. 10. The dashed line represents a power-law fit with cancellation exponent $\chi=0.127$, while the dotted curve has cancellation exponent unity, representing the behavior of random noise. We thus again find a cancellation exponent that is half as large as that of Pietarila Graham et al. (2009), and approximately the same as we found from our SP analysis based on our conversion of polarization to flux density that accounts for the intrinsic properties of the collapsed flux elements. This agreement supports the validity of this smaller cancellation exponent.

The observed $V$ maps represent the superposition of "true" $V$ and noise. Since random noise has a very steep cancellation function (with exponent unity), the noise contribution, even if it would dominate at the Hinode scale, would quickly decline to become insignificant with increasing scale size. The composite cancellation function would then be steep at small scales but quickly approach the more gradual slope of the noise-free cancellation function. The circumstance that the empirical cancellation function has a consistent slope throughout practically the whole range of scales in Fig. 10 is evidence that the influence of random noise is insignificant, in spite of the substantially lower $\mathrm{S} / \mathrm{N}$ ratio for the FG as compared with the SP data. The random noise is suppressed by using sufficient statistics with a large number of magnetograms.

In addition to random noise that mainly comes from the finite photon statistics there can in principle also be systematic noise that has correlations at different spatial scales, for instance due to non-flatness of the zero level or the gain table for Stokes $V$. Any such spurious effects would tend to steepen the cancellation function. The smaller value of our cancellation exponent is an indication that it is not compromised by such effects.

\section{Sizes and field strengths of collapsed flux elements}

\subsection{Derivation of size distributions}

There is a continuous range of $V$ polarization amplitudes in the top panel of Fig. 8 because there is a continuous range of filling factors for the collapsed flux population, from close to zero to values of about $60 \%$. In contrast the intrinsic field strength varies much less with polarization amplitude (since the $V$ line ratio is a slow function of $V$ ). In Stenflo (2010) a calibrated relation between the $V$ amplitude and the intrinsic field strength $B_{\mathrm{f}}$ of the flux tube component was given, and a procedure to obtain the vertical flux density $B_{\mathrm{v}}$ from $V$ and the inclination angles was described in detail. The angular distribution of field vectors was represented by the function $\mu^{a}$, where $\mu=\cos \gamma$, and $\gamma$ is the inclination angle. The exponent $a$ that characterizes the distribution is a function of flux density and was determined from a combination of the observed $Q, U$, and $V$ amplitudes. The $a$ function allows us to assign an average value $\bar{\mu}$ for $\cos \gamma$ for each $B_{\mathrm{v}}$ bin. In the following we will for approximate, statistical purposes connect the total flux density $B$ with the vertical flux density $B_{\mathrm{v}}$ through the relation $B_{\mathrm{v}}=B \bar{\mu}$. Since $\mu$ is not uncorrelated with $B$ and $B_{\mathrm{v}}$ this statistical relation is only approximate, but it is good enough for our purposes, in particular since the angular distribution is peaked around the vertical direction and therefore $\mu$ is close to unity except in the region of small flux densities, in which the distribution becomes increasingly isotropic. For an isotropic distribution $\bar{\mu}=1 / 2$.

Since the angular distributions that we use here contradict earlier claims that there is a predominance of horizontal magnetic flux on the quiet Sun, we have in the Appendix tried to clarify the nature of the trouble with the horizontal fields.

With the $B_{\mathrm{f}}, B_{\mathrm{v}}$, and $\bar{\mu}$ that we can thus assign to each $V$ amplitude we obtain the filling factor $f$ through

$f=\frac{B_{\mathrm{v}}}{B_{\mathrm{f}} \bar{\mu}}$.

Assuming that the filling factor is due to a single flux element inside the Hinode $200 \mathrm{~km}$ spatial resolution element, the size $d_{\mathrm{f}}$ of that flux element is

$d_{\mathrm{f}}=200 \sqrt{f} \mathrm{~km}$.

If the filling factor is instead represented by a cluster of several elements rather than by a single flux tube, then the size of each flux element is correspondingly smaller. Eq. (5) can therefore be seen as an upper limit to the flux tube size.

From the analysis in Stenflo (2010) we know that the collapsed flux component dominates for all $V$ amplitudes above $0.5 \%$ (as shown in Fig. 10 of that paper). For smaller polarizations the distinction between the collapsed and uncollapsed populations becomes undefined because of excessive influence of noise. From the same analysis it also follows that $V=0.5 \%$ corresponds to $B_{\mathrm{v}} / \bar{\mu}=50 \mathrm{G}$, while $B_{\mathrm{f}}=840 \mathrm{G}$. The filling factor $f$ is therefore $50 / 840=6 \%$, which gives a flux tube size of about $50 \mathrm{~km}$. Since it is most likely that the collapsed flux population continues to contribute significantly also for $V$ amplitudes below $0.5 \%$, we expect an abundance of strong-field flux tubes with still smaller sizes.

We can go a step further and use the observed PDF (probability density function) for the vertical flux densities $P_{\mathrm{v}} \sim \mathrm{d} N / \mathrm{d} B_{\mathrm{v}}$, the histogram of the number of pixels per bin in $B_{\mathrm{v}}$. If $r_{w}$ is the relative abundance of the weak or uncollapsed flux population as given in Fig. 8 of Stenflo (2010), then the PDF $P_{d}$ for the flux tube sizes is given by

$P_{d}=\left(1-r_{w}\right) P_{\mathrm{v}} \frac{\mathrm{d} B_{\mathrm{v}}}{\mathrm{d} d_{\mathrm{f}}}$.

Via the previously mentioned relations that we have found between $V, B_{\mathrm{f}}, B_{\mathrm{v}}, f$, and $d_{\mathrm{f}}$, the derivative $\mathrm{d} B_{\mathrm{v}} / \mathrm{d} d_{\mathrm{f}}$ can be calculated. The function for $r_{w}$ is extracted from Fig. 8 of Stenflo (2010), where for the $V$ range $0-0.5 \%$ we use the two alternative extrapolations for $r_{w}$. With one extrapolation (the physically more plausible one) $r_{w}$ increases steeply to reach $100 \%$ in the limit of vanishing polarization, which implies that in the weak flux density limit all flux is uncollapsed. With the other smooth extrapolation $r_{w}$ only reaches $37 \%$ at $V=0$. With these functions it then becomes possible to convert $P_{\mathrm{v}}$ for the vertical flux densities into histograms $P_{d}$ for the flux tube sizes.

For this conversion we will for $P_{\mathrm{v}}$ use the symmetric part of the analytical representation of the observed PDF, as given by Eqs. (6)-(10) in Stenflo (2010). It contains a strongly peaked core that is represented by a stretched exponential (after the Gaussian broadening due to measurement noise has been removed) and Lorentzian wings that decline quadratically.

The distributions $P_{d}$ for the flux tube sizes, which represent histograms $\mathrm{d} N / \mathrm{d} d_{\mathrm{f}}$, can be converted to histograms $\mathrm{d} N / \mathrm{d}\left(\log d_{\mathrm{f}}\right)$ with logarithmic binning in $d_{\mathrm{f}}$, simply by using $d_{\mathrm{f}} P_{d}$ instead. In Fig. 11 we show the amplitude-normalized PDFs with logarithmic binning for the two choices of described extrapolations of $r_{w}$ in the $V$ range $0-0.5 \%$. The solid curve represents the physically more plausible extrapolation, for which all flux is uncollapsed in the limit of vanishing flux density, while the dashed curve represents the other extrapolation. Since this other extrapolation 


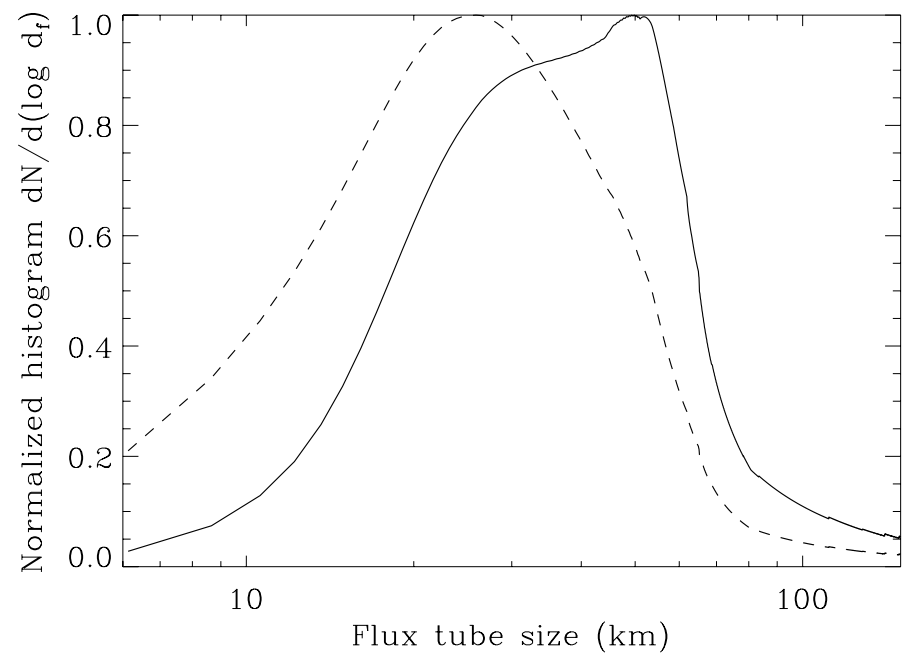

Fig. 11. Histograms with logarithmic binning of flux tube sizes, derived from the established statistical relations between Stokes $V$, intrinsic field strengths, flux densities, and the relative abundances of the collapsed and uncollapsed flux populations. The solid and dashed curves are based on two different extrapolations of the relative abundance of the uncollapsed flux population in the limit of small flux densities, as described in the text.

gives much more weight to the collapsed population in the limit of small flux densities, which corresponds to small sizes, it has the effect of pushing the whole distribution towards the smaller sizes, although the shift is not that dramatic and is smaller than the width of the distributions. The maximum of the solid curve is at about $50 \mathrm{~km}$, the dashed curve at $26 \mathrm{~km}$. Due to the different choices of $r_{w}$ for the two curves their area normalizations become different, so when using a common amplitude normalization the two curves appear different for all sizes. However, the relative shapes of the two curves are in fact nearly identical for $d_{\mathrm{f}}>60 \mathrm{~km}$, the region that corresponds to a common $r_{w}$ function.

From these histograms we see that the great majority of flux tubes have sizes in the range $10-70 \mathrm{~km}$. This however does not imply that all of them have $\mathrm{kG}$ field strengths. As we have seen from Eqs. (4) and (5) and the accompanying discussion, we can for each observed $V$ statistically associate (for the collapsed population) an intrinsic field strength $B_{\mathrm{f}}$ and a flux tube size $d_{\mathrm{f}}$. The resulting plot of $B_{\mathrm{f}}$ vs. $d_{\mathrm{f}}$ is shown in Fig. 12. The field strength drops from about 1600 to about $850 \mathrm{G}$ as we go from flux tube size 150 down to $50 \mathrm{~km}$. We are presently not able to continue this relation down to still smaller scales, since these scales correspond to Stokes $V$ amplitudes that are too small $(<0.5 \%)$ for a reliable determination of the $V$ line ratio, which is the basis for the determination of $B_{\mathrm{f}}$. The slope of the $B_{\mathrm{f}}-d_{\mathrm{f}}$ relation in Fig. 12 indicates that the intrinsic field strength will quickly drop down in the $\mathrm{hG}$ (hectogauss) domain as we move down in size below $50 \mathrm{~km}$, so that when one reaches the $10 \mathrm{~km}$ scale the distinction between intrinsically strong and weak fields will no more be well defined.

\subsection{Physical interpretation}

Although our use of the term "collapsed flux" does not necessarily imply that the bundled, strong-field flux elements are formed by the mechanism of convective collapse (Parker 1978; Spruit 1979; Spruit \& Zweibel 1979; Unno \& Ando 1979), this mechanism remains the favored one, since it provides a way for the flux

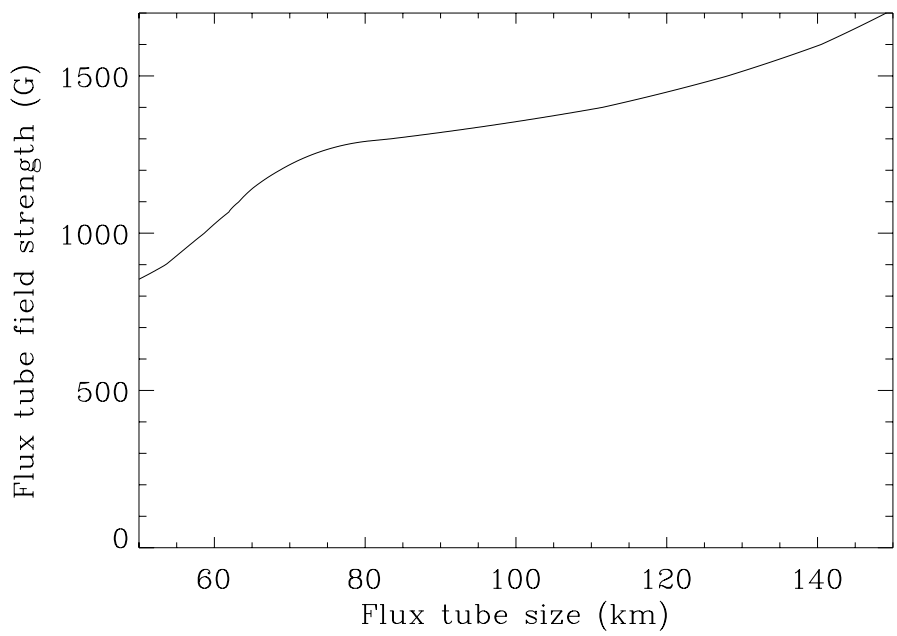

Fig. 12. Statistical relation for the intrinsic field strength $B_{\mathrm{f}}$ of the flux tubes as a function of their sizes $d_{\mathrm{f}}$. Below a scale of $50 \mathrm{~km}$ the relation cannot presently be determined because the corresponding Stokes $V$ line ratio becomes noise dominated.

to spontaneously and quickly end up in flux bundles with a rather well-defined field strength, when the internal magnetic pressure of the largely evacuated interior balances the well-defined external gas pressure. This instability is most efficient in the domain where the superadiabatic gradient is large and the collapsing region of predominantly vertical flux is optically thick in the horizontal direction. The reason is that the instability is driven by the process of adiabatic cooling. An initially weak downdraft inside the flux region establishes an internal adiabatic temperature gradient if the flow is thermally shielded from the surroundings on the time scale of the flow, which is the case if the photon mean free path in the horizontal direction is much smaller than the flux region itself (criterion of optical thickness). Since the outside temperature gradient is superadiabatic the flow generates a temperature deficit inside the flux region (at equal geometrical depth) plus a partial evacuation (reduction of the gas density), which both lead to a pressure deficit and therefore a collapse with an amplification of the downflow. The instability saturates when an inside magnetic pressure has been produced that can, together with the remaining internal gas pressure, balance the external gas pressure and thereby stop further collapse.

In Fig. 13 we have used the atmospheric model of Maltby et al. (1986) and convection zone model of Spruit (1977) to derive how the photon mean free path (in the continuum at $5000 \AA$ ) and superadiabatic gradient (difference between the magnitudes of the actual logarithmic temperature gradient and the corresponding adiabatic temperature gradient) vary with height in the atmosphere and immediately below. The zero point of the geometrical height scale is where the optical depth in the continuum at $5000 \AA$ is unity.

We notice in Fig. 13 that the superadiabaticity has a welldefined peak just below the photosphere, around a height of $-40 \mathrm{~km}$, but rapidly drops to zero as we enter the lower photosphere. This drop is what marks the end of the convection zone, since the convective instability requires a finite superadiabatic gradient. The solar granulation that we observe in the photosphere is therefore mainly overshoot from the underlying convection zone. At the location of the superadiabaticity peak the photon mean free path is $10 \mathrm{~km}$, but it increases rapidly to become about $70 \mathrm{~km}$ at the point where the convection zone formally ends. 


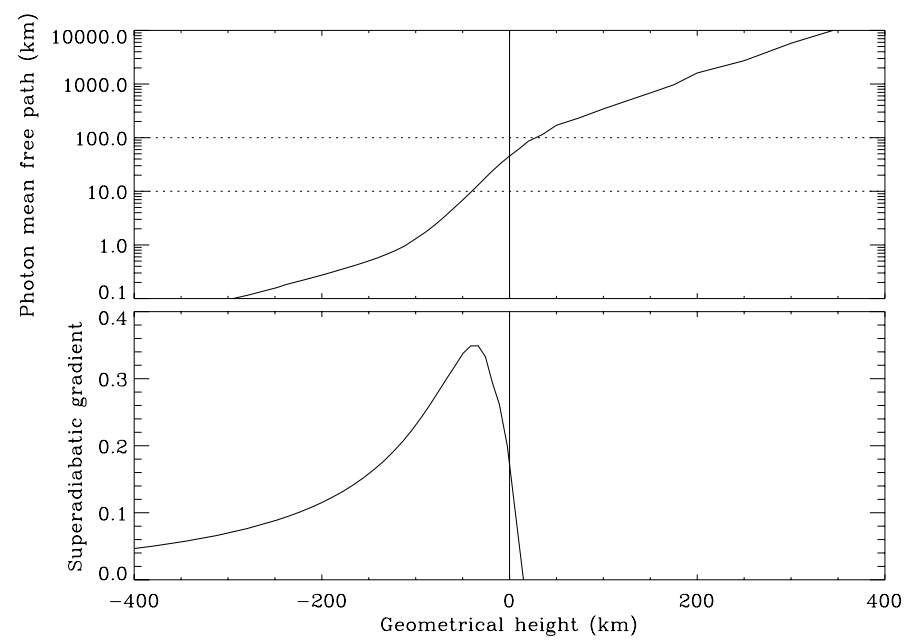

Fig. 13. Top panel: photon mean free path. The dotted horizontal lines mark the 10 and $100 \mathrm{~km}$ scales for easy reference. Bottom panel: superadiabatic gradient. The zero point of the geometrical height scale is at optical depth unity of the continuous spectrum at $5000 \AA$.

As we go further down below the superadiabaticity peak the photon mean free path rapidly decreases to $1 \mathrm{~km}$ and below, allowing in principle collapsed structures with correspondingly small sizes to be formed, but as they expand with height with the drop in external gas pressure, their sizes will be substantially larger when they emerge into the photosphere. For these reasons the empirically determined range of $10-70 \mathrm{~km}$ that we have found from the distributions in Fig. 11 is the range that one may theoretically expect from the convective collapse mechanism.

As the requirement of lateral optical thickness only introduces a lower size limit, it could be argued that there is no upper limit, the distributions may extend to any larger size. This is true, and we do indeed see flux tubes over a broad range of larger sizes, like sunspots (although the convective collapse mechanism may not be relevant to sunspot formation). However, these larger flux tubes are generally only seen in localized, special areas on the Sun (active regions) that are directly governed by the global dynamo operating deep inside the Sun. Here we are dealing with the ubiquitous magnetoconvection in the near surface layers of the quiet Sun. Any larger-size flux tube is subject to fragmentation into smaller pieces by the fluting instability, until the optically thin domain is reached, where the strong field cannot be maintained by recollapse. The weakened field will then either be tangled up by the turbulent motions or merge with other flux elements to form larger entities that satisfy the conditions for recollapse. The combined effect of all these fragmentation and recollapse processes would tend to be that the flux tube size spectrum piles up near the region where the transition between the optically thick and thin regimes occurs. It is also natural that with the fragmentation, and as we approach the optically thin regime, the intrinsic field strength will decline. This scenario is a description in words of the apparent meaning of Figs. 11-13.

\section{Properties of the hidden flux}

The cancellation function that was given in Fig. 10 shows that there exists magnetic flux that is presently hidden because the contributions from the mixed, opposite polarities cancel out due to the spatial smoothing by the finite resolution. More of this flux will become visible as we improve the resolution of our telescopes. The more we are allowed to extrapolate the cancellation function into the unresolved domain, the more hidden flux there is. To know how far we may extrapolate we need some additional constraint. This comes from the Hanle effect, which determines an absolute level that should be reached by the extrapolation.

To understand this better we need to remember that the Zeeman-effect measurements contained in the cancellation function refer to flux densities (average of the field over the resolution element) and therefore depend on the resolution scale that is considered. In contrast the Hanle effect provides information not on the flux densities but on the field strengths of the hidden field. This is a resolution-independent quantity, it is the flux density in the limit of infinite resolution. This means that the cancellation function should be extrapolated until its flux densities become equal to the field strength that is demanded by the Hanle effect. The spatial scale at which this field strength is reached will then represent the characteristic scale of the elements that are the carriers of most of the hidden flux.

We will next try to combine our cancellation function with the Hanle constraint to estimate the amount of hidden flux and its characteristic spatial scale. There are of course many poorly known factors that cause great uncertainties in these estimates. One problem is that the Hanle depolarization constraint is not just satisfied by a single field strength but by a variety of different probability density functions for the field strength distribution. Another problem is that the Hanle analysis is very sensitive to the correct treatment of the collisional depolarization effects and macroturbulence in the solar atmosphere. Here we will try to clarify how these effects enter so that we can better appreciate the degree of validity of the results and see what is needed to improve them.

The most thorough modeling to date of the Hanle depolarization effect is by Trujillo Bueno et al. (2004), who did polarized radiative transfer calculations in 3D atmospheres generated by numerical simulation of magnetoconvection to model the Hanle depolarization effect in the Sr I $4607 \AA$ line using data sets from Stenflo et al. (1997), Trujillo Bueno et al. (2001), and Bommier \& Molodij (2002). When a single-valued field with an isotropic distribution of field vectors was assumed, a field strength of $60 \mathrm{G}$ was found. If on the other hand an exponentially shaped PDF for the field strengths was assumed (in combination with the assumption of an isotropic angular distribution for all field strengths), the average field strength of the PDF was found to be $130 \mathrm{G}$. This illustrates the sensitivity of the interpretation to the assumption used for the underlying PDF (which is not a direct observable, since it is made up of spatially unresolved fields). Nevertheless, any PDF with a shape that declines monotonically from smaller to larger field strengths will give average field strengths larger than with the single-valued assumption.

\subsection{Relative importance of the PDF core and tail regions}

In our analysis of the Hinode data we have determined not only detailed PDF shapes but also angular distributions (which in general are not isotropic). Although these distributions are resolution dependent and refer to the Hinode $200 \mathrm{~km}$ scale size, let us next explore which portions of the PDF contribute to the observed Hanle depolarization and which portions govern the average flux densities. As we will see the Hanle depolarization is mainly determined by the inner core of the PDF, while the extended tails have a large influence on the average flux densities of the distribution.

The Hanle depolarization factor $k_{\mathrm{H}}$ by which the observed linear scattering polarization is reduced due to the presence of 
a hidden, tangled field of strength $B$ and with an angular distribution given by $\mu^{a}$ (which we used in Sect. 5 above) is (Stenflo 1987)

$k_{\mathrm{H}}=1-\frac{6}{(a+3)(a+5)}\left[(a+1) \sin ^{2} \alpha_{1}+\sin ^{2} \alpha_{2}\right]$,

where $a$ is the exponent determining the angular distribution. $a=0$ represents an isotropic distribution, which we have in the limit of small flux densities, while larger values of $a$ represent distributions that are peaked around the vertical direction (which is the case for large flux densities). In the limit of infinite $a$ (strictly vertical fields) there is no depolarization $\left(k_{\mathrm{H}}=1\right)$. In the saturated strong-field limit of an isotropic distribution $k_{\mathrm{H}}=0.2$.

The Hanle angles $\alpha_{1}$ and $\alpha_{2}$ are determined by (Stenflo 1994)

$\tan \alpha_{q}=q k_{\mathrm{c}}^{(2)} B / B_{0}$,

where $B_{0}$ is the field strength for which the Larmor precession rate (scaled with the Landé factor) equals the spontaneous decay rate (or inverse life time) of the excited atomic state.

$k_{\mathrm{c}}^{(K)}=\frac{\gamma_{\mathrm{N}}}{\gamma_{\mathrm{N}}+\gamma_{\mathrm{c}}^{(K)}}$

is the branching ratio for the collisional depolarization, with $\gamma_{\mathrm{N}}$ being the natural, spontaneous decay rate and $\gamma_{\mathrm{c}}^{(K)}$ being the rate of collisional destruction of the $2 K$-multipole of the excited state (Stenflo 1994).

From these equations follows a very important property: the effective field-strength range of Hanle sensitivity is not just given by $B_{0}$ (which only depends on known atomic physics and has nothing to do with any model atmosphere), but by its "effective" value

$B_{0, \mathrm{eff}}=B_{0} / k_{\mathrm{c}}^{(2)}$

(note that $\gamma_{\mathrm{c}}^{(2)} \approx \gamma_{\mathrm{c}} / 2$, where $\gamma_{\mathrm{c}}$ is the elastic collision rate). It means that the accuracy of the field-strength determinations much depends on the accurate treatment of the collisional effects in the solar atmosphere, because they govern the field-strength scale (cf. Stenflo 1982). In the photosphere the collisional rates generally dominate over the spontaneous decay rates and depend in a sensitive way on the chosen atmospheric model.

For the Sr I $4607 \AA$ line we find from atomic physics that $B_{0} \approx 23 \mathrm{G}$. The result of Trujillo Bueno et al. (2004) that the observed depolarization corresponded both to a field strength of $60 \mathrm{G}$ when an isotropic singe-valued ( $\delta$ function PDF) field was assumed and to an average field of $130 \mathrm{G}$ when an exponential

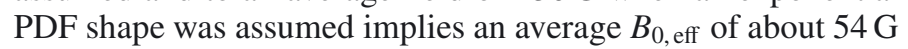
rather than the $23 \mathrm{G}$ for the collision-free case. Any other value of $B_{0, \text { eff }}$ would not give the combination of 60 and $130 \mathrm{G}$ for the two PDFs used. This implies a collisional branching ratio of $k_{\mathrm{c}}^{(2)} \approx 0.42$, which means that $\gamma_{\mathrm{c}}^{(2)} / \gamma_{\mathrm{N}} \approx 1.4$. We give these numbers here simply to illustrate the crucial role that the collisional depolarization rates play in determining the whole field-strength scale in the conversion of observed Hanle depolarization into field strength.

Another major difficulty in the Hanle analysis is that the socalled "observed" Hanle depolarization factor $k_{\mathrm{H}}$ is not a direct observable but also heavily depends on radiative-transfer modeling. $k_{\mathrm{H}}$ represents the ratio between two quantities: (1) the observed scattering polarization, and (2) the value of this polarization in the non-magnetic case. While the first quantity is directly observed, the second quantity is determined via polarized radiative transfer. The reason why Trujillo Bueno et al. (2004) obtained substantially higher field strengths than previously reported did not lie in the calculation of the collisional effects, but in the different treatment of macroturbulence, which significantly affects the derivation of the non-magnetic polarization. The use of 3D model atmospheres produced by numerical simulations allowed Trujillo Bueno et al. (2004) to avoid the use of the macroturbulent concept and thereby suppress this source of error.

Next we will use the PDF for the flux densities and the angular distributions determined from the Hinode observations to determine which parts of the PDF govern the apparent Hanle field strength and the average field strength. For the PDF of the vertical flux densities, $P_{\mathrm{v}}$, we choose the analytical representation of the symmetric part of the PDF as specified in Stenflo (2010), as we did before in Sect. 5. It is given by the dotted curve in the top panel of Fig. 14. $P_{\mathrm{v}}$ can in our treatment be seen as a superposition of three components:

$P_{\mathrm{v}}=P_{v, \mathrm{unc}}+P_{v, \mathrm{ft}}+P_{v, \mathrm{~nm}}$.

$P_{v \text {, unc }}$ represents the contribution from the uncollapsed flux population, $P_{v \text {,ft }}$ the contribution from the magnetic (flux tube) component of the collapsed population, while $P_{v, \mathrm{~nm}}$ accounts for the non-magnetic component of the collapsed population. Although this "non-magnetic" component formally has zero field strength and therefore contributes with a PDF in the form of a $\delta$ function peak at zero $G$, it must be accounted for, since it represents a major fraction of the solar surface. The $\delta$ function shape of the PDF is of course an artefact of the 2-component idealization, so to understand its real nature we must go beyond such idealizations. This issue will be discussed in greater detail in Sect. 7.

If we as before (in Sect. 5) let $r_{w}$ represent the relative abundance of the weak-field, uncollapsed population, then

$$
\begin{aligned}
P_{v, \text { unc }} & =r_{w} P_{\mathrm{v}}, \\
P_{v, \mathrm{ft}} & =\left(1-r_{w}\right) f P_{\mathrm{v}}, \\
P_{v, \mathrm{~nm}} & =\left(1-r_{w}\right)(1-f) P_{\mathrm{v}} .
\end{aligned}
$$

As mentioned in Sect. 5 a statistical estimate of the total flux density $B$ may be obtained from the statistically approximate relation $B=B_{\mathrm{v}} / \bar{\mu}$, where $\bar{\mu}$ is the average of $\cos \gamma$ for a given value of $B_{\mathrm{v}}$. For the uncollapsed, weak flux population we will assume that $B$ represents not only the flux density but also the field strength. In contrast, for the collapsed population the field strengths of the two components are $B_{\mathrm{f}}$ and zero, and $B=f B_{\mathrm{f}}$, where $f$ is the filling factor. $r_{w}, f, P_{\mathrm{v}}, B, B_{\mathrm{f}}$, and $a$ (which describes the shape of the angular distribution) are known functions of $B_{\mathrm{v}}$ (from the empirical determinations in Stenflo 2010).

The Hanle depolarization factor $k_{\mathrm{H}}$ of Eq. (7) is a function of both the angular distribution (represented by parameter $a$ ), and field strength $B$ or $B_{\mathrm{f}}$. Note that there is no direct relation with the flux density, one has to use the field strength. The average of $k_{\mathrm{H}}$ for the given field distribution is then

$$
\begin{aligned}
\left\langle k_{\mathrm{H}}\right\rangle= & {\left[\int k_{\mathrm{H}}(a, B) P_{v, \text { unc }} \mathrm{d} B_{\mathrm{v}}+\int k_{\mathrm{H}}\left(a, B_{\mathrm{f}}\right) P_{v, \mathrm{ft}} \mathrm{d} B_{\mathrm{v}}\right.} \\
& \left.+\int k_{\mathrm{H}}(a, 0) P_{v, \mathrm{~nm}} \mathrm{~d} B_{\mathrm{v}}\right] / \int P_{\mathrm{v}} \mathrm{d} B_{\mathrm{v}} .
\end{aligned}
$$

Note that $k_{\mathrm{H}}(a, 0)=1$ for the non-magnetic component (for any value of $a$ ). In contrast, for the average vertical and total flux densities we may simply use

$$
\begin{aligned}
\left\langle B_{\mathrm{v}}\right\rangle & =\int B_{\mathrm{v}} P_{\mathrm{v}} \mathrm{d} B_{\mathrm{v}} / \int P_{\mathrm{v}} \mathrm{d} B_{\mathrm{v}}, \\
\langle B\rangle & =\int B P_{B} \mathrm{~d} B / \int P_{B} \mathrm{~d} B,
\end{aligned}
$$



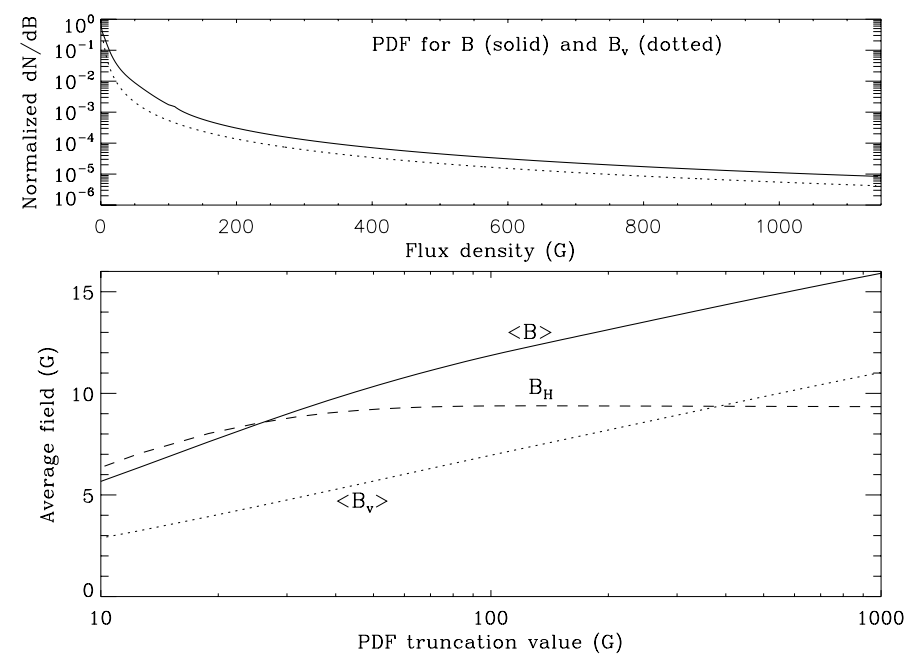

Fig. 14. The top panel shows analytical representations of empirically determined PDFs for the vertical flux density $B_{\mathrm{v}}$ and the total flux density $B$ that are used for the calculations of the average flux densities $\left\langle B_{\mathrm{v}}\right\rangle$ and $\langle B\rangle$ in the bottom panel. These averages are given as functions of the maximum flux density (truncation value) allowed in the integration over the PDF. $B_{\mathrm{H}}$ is the field strength derived from the Hanle depolarization, assuming a single-valued field with an isotropic angular distribution.

where $P_{B}$ for the total flux densities $B$ is

$P_{B}=P_{\mathrm{v}} \frac{\mathrm{d} B_{\mathrm{v}}}{\mathrm{d} B}$

and is represented by the solid curve in the top panel of Fig. 14.

The integrations in these equations are done from $B=0$ to an upper limit $B=B_{\text {trunc }}$, where $B_{\text {trunc }}$ is the maximum value to which we let the PDF tail extend. We will next treat this maximum as a variable truncation value, to explore the relative importance of the core and tail regions of the PDF for the mean values and for the Hanle effect.

The solid and dotted curves in the bottom panel of Fig. 14 show how the average values of $B$ and $B_{\mathrm{v}}$ as determined by Eq. (14) depend on the truncation value $B_{\text {trunc }}$. We see that the extended PDF tails, where the PDF is $<1 \%$ of its maximum value, contribute about as much as the inner, heavily populated core region of the PDF.

Let us now take the average value $\left\langle k_{\mathrm{H}}\right\rangle$ of the Hanle depolarization and convert it to a "Hanle field strength" $B_{\mathrm{H}}$ based on the assumption that the field is single-valued with an isotropic angular distribution, assuming that the parameter that determines the field-strength sensitivity for the Hanle effect is $B_{0 \text {, eff }}=54 \mathrm{G}$, the value that was implicitly used in the Sr I $4607 \AA$ analysis of Trujillo Bueno et al. (2004). We then obtain the dashed curve in Fig. 14. $B_{\mathrm{H}}$ saturates at a value of about $9 \mathrm{G}$ already for PDF truncation values as low as about $30 \mathrm{G}$. This shows that the PDF tails have almost no influence on the Hanle field strength $B_{\mathrm{H}}$, it is almost exclusively determined by the PDF core region.

There are several reasons why the PDF tails are unimportant for $B_{\mathrm{H}}$ in our case. The tails are dominated by the collapsed flux population, which contribute little to the Hanle effect because: (1) the field distribution is peaked around the vertical direction, and the Hanle effect vanishes for vertically oriented fields. (2) The magnetic filling factor is small, and the Hanle effect scales with the filling factor. (3) The field strengths of the strong magnetic component lie in the fully saturated Hanle regime, in which the Hanle effect does not depend any more on field strength. (4) Our PDF has a strongly peaked core region, so that the inner

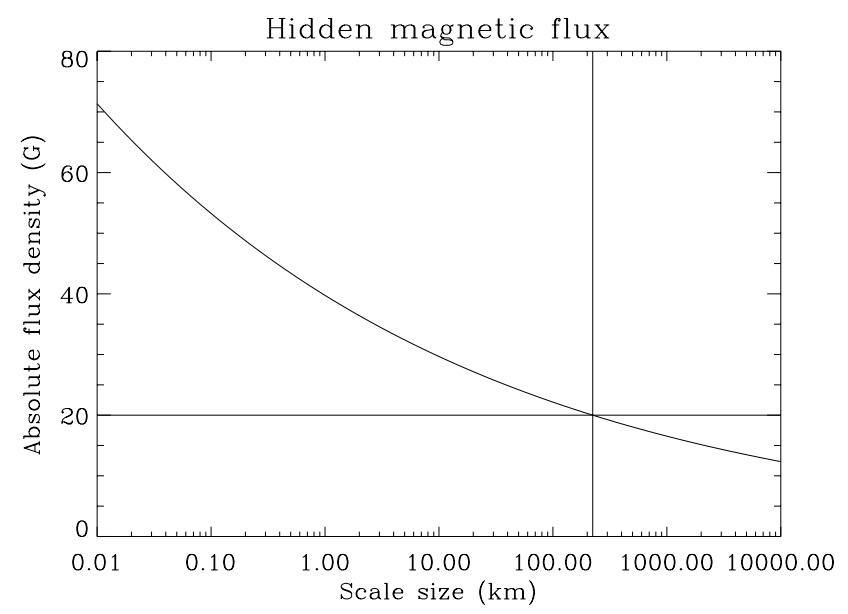

Fig. 15. The power-law cancellation function of Fig. 10 (the dashed curve of that figure), extrapolated down to the magnetic diffusion scale of $10 \mathrm{~m}$. Here it has been normalized to a value of $20 \mathrm{G}$ at the Hinode resolution scale (marked by the vertical solid line), to make the scale represent the average of the total flux densities.

parts of the tails are much more suppressed than for instance for an exponentially-shaped PDF. In comparison Trujillo Bueno et al. (2004) not only used an exponentially shaped PDF, but they also assumed that the angular distribution would remain isotropic for all field strengths. In this case there will be a strong influence on the Hanle effect also for fields of order $100 \mathrm{G}$.

\subsection{Extrapolation of the cancellation function}

With this background we can better appreciate the potential, difficulties, and uncertainties in trying to determine the properties of the hidden magnetic fields from the constraints of the Hanle effect. In Fig. 15 we have taken the $d^{-0.127}$ power law of the cancellation function that was given in Fig. 10 and extrapolated it down to the magnetic diffusion scale of $10 \mathrm{~m}$, where the turbulent structuring of the field is expected to end. Now the cancellation function is given with respect to a total (rather than vertical) flux density scale and has been normalized to a value of $20 \mathrm{G}$ at the Hinode resolution scale. This is the average value of the total flux density that we find in the Hinode analysis of Stenflo (2010). It might be slightly overestimated, since it is somewhat larger than what comes out of the analytical PDF representation in Fig. 14, from which the noise contribution has been removed to first order. Such details are however unimportant in this context, since the level to be reached according to the Hanle analysis of Trujillo Bueno et al. (2004) is at least 3 times larger $(60 \mathrm{G})$. Furthermore, the Hanle analysis that is here only based on the fields that are not hidden on the Hinode scale gives field strengths of order $10 \mathrm{G}$ according to $B_{\mathrm{H}}$ in Fig. 14, smaller by a factor of 6 than the $60 \mathrm{G}$ value. This is a huge gap to be bridged. It indicates that the hidden flux elements must be far smaller, by orders of magnitude, than the currently best telescope resolution. To bridge such a gap with the present cancellation function one would need to extrapolate it all the way down to the region of 10$100 \mathrm{~m}$ close to the diffusion limit at the end of the scale spectrum (and possibly even further, if it were physically allowed).

While keeping in mind the uncertainties of all these estimates and extrapolations, the combination of the currently available empirical constraints seem to suggest that most of the hidden flux quickly cascades down to the range near the end of the scale spectrum and piles up there. However, such conclusions 
also depend on shaky assumptions of fractal-like self-similarity that allow us to extrapolate a cancellation function with a fixed cancellation exponent. In the next section we will explore the underlying nature of the spatially unresolved fields by examining the magnetic dichotomy in terms of the PDF, to gain insight into the origin of the dichotomy and reveal properties that may cause the dichotomy to disappear at a deeper level in the spatially unresolved domain.

\section{Origin of the magnetic dichotomy}

The PDFs in the top panel of Fig. 14 are smooth functions that connect the smallest and the largest gauss values in a continuous way. There is no hint of any dichotomy, since the displayed PDFs represent flux densities, not field strengths. To see the dichotomy in the field distribution, we have to convert $P_{v \text {, unc }}, P_{v \text {, ft }}$, and $P_{v \text {, nm }}$ in Eq. (13) from flux density distributions to field strength distributions.

If we in the case of the uncollapsed flux population assume that the fields are intrinsically weak and resolved, so that we can equate the field strength with the total flux density $B$, then we obtain the PDF for the field strengths through division of $P_{\mathrm{v}}$ by $\mathrm{d} B / \mathrm{d} B_{\mathrm{v}}$, as in Eq. (14). The validity of this crude assumption will be discussed later, but for the moment we hardly have any other choice than to use it. Similarly, for the magnetic component of the collapsed population we find the field-strength PDF through division of $P_{\mathrm{v}}$ by $\mathrm{d} B_{\mathrm{f}} / \mathrm{d} B_{\mathrm{v}}$, assuming that we "know" the intrinsic field strength $B_{\mathrm{f}}$ from the Stokes $V$ line ratio, which gives a statistical relation between $B_{\mathrm{f}}$ and $B_{\mathrm{v}}$.

The situation is different for the non-magnetic component of the collapsed population. Since it has an "intrinsic field strength" of zero G, it would contribute to a field-strength PDF that is a $\delta$ function peak placed at zero G. As much of the solar surface is occupied by this component, it must be accounted for. However, since its non-magnetic nature is due to an unphysical idealization that was introduced for the 2-component model with the purpose of extracting intrinsic field strengths for the magnetic component, it is obvious that the $\delta$ function shape is an artefact of the chosen idealization. We would rather expect that the "non-magnetic" component is physically related to the uncollapsed flux. However, in contrast to the uncollapsed flux population revealed by the line-ratio analysis, it may represent hidden uncollapsed flux that does not contribute significantly to the net flux through the spatial resolution element, since the net flux is dominated by the magnetic component of the collapsed population.

Due to its probable physical relationship with the partially resolved uncollapsed flux, we will for representational purposes here assume that the "non-magnetic" component contributes with a PDF that has the same shape as that of the uncollapsed flux. In this case it simply enters as a scaling factor $s$ for the PDF contribution from the uncollapsed flux:

$s=1+\frac{\int P_{v, \mathrm{~nm}} \mathrm{~d} B_{\mathrm{v}}}{\int P_{v, \text { unc }} \mathrm{d} B_{\mathrm{v}}}$.

For our data set with the assumption that the relative abundance $r_{w}$ of the uncollapsed population becomes $100 \%$ at $B_{\mathrm{v}}=0$, we get $s=1.24$. The reason why this does not differ that much from unity is that $P_{\mathrm{v}}$ is extremely peaked at zero G, where most of the uncollapsed flux comes from, while the "non-magnetic" component has its source in the pixels that represent the collapsed flux.

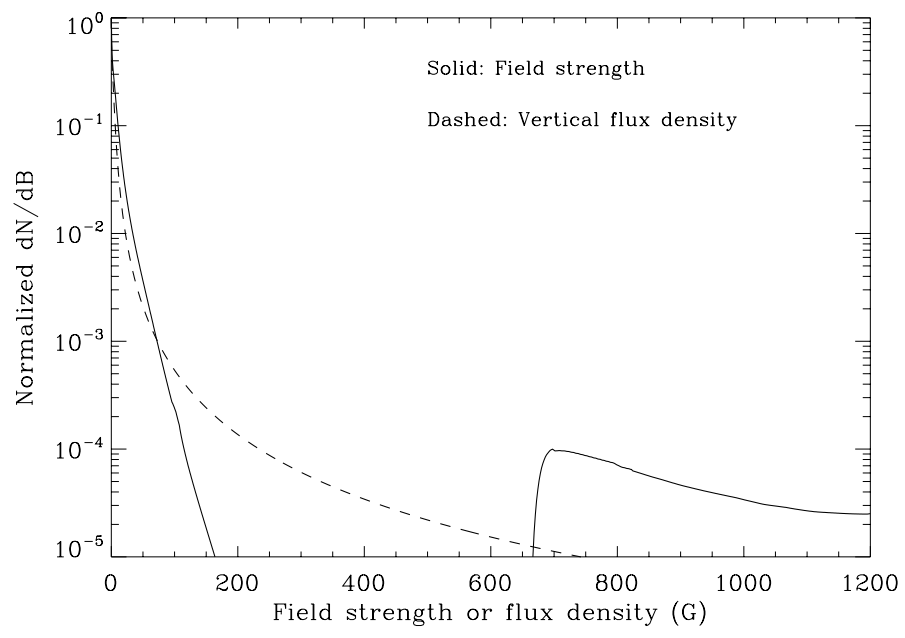

Fig. 16. Solid curve: PDF for the intrinsic field strengths, derived from the PDF for the vertical flux densities, $P_{\mathrm{v}}$ (dashed curve). While the flux density PDF connects the low and high field values in a continous way, the field-strength distribution exhibits an apparent dichotomy with a gap, the nature of which is discussed in the text.

With these various rather reasonable assumptions we can express the composite PDF $P_{\text {intrinsic }}$ for the "intrinsic" (of course heavily model-dependent) field strengths as

$P_{\text {intrinsic }}=s P_{v, \text { unc }} / \frac{\mathrm{d} B}{\mathrm{~d} B_{\mathrm{v}}}+P_{v, \mathrm{ft}} / \frac{\mathrm{d} B_{\mathrm{f}}}{\mathrm{d} B_{\mathrm{v}}}$.

The result is plotted as the solid curve in Fig. 16. For comparison we have plotted as the dashed curve $P_{\mathrm{v}}$ for the vertical flux densities, which serves as the basis for the derivation of the solid curve.

A striking feature of Fig. 16 is the dichotomy exhibited by the PDF of the intrinsic field strengths, with two disjunct regions, one strong-field branch belonging to the magnetic component of the collapsed flux population, and one weak-field branch, associated with the uncollapsed population and the "non-magnetic" component. For the flux density PDF such a dichotomy is not seen, since the field strengths get multiplied by a filling factor that has a continuous range of values from 0 towards (but not reaching) 1, which causes the flux densities to be redistributed over the whole range in a smoothed-out way.

The existence of such a dichotomy has not been demonstrated in numerical simulations of magnetoconvection. The question therefore becomes whether or not there is a real or only apparent contradiction with the simulations. Although no definite answer to this question is possible at the present time, there are good reasons to suspect that the apparent huge gap in the PDF between 150 and $670 \mathrm{G}$ may be an artefact of the finiteness of the instrument systems (finite $\mathrm{S} / \mathrm{N}$ ratio, finite resolution), which causes truncation-like effects that we will try to clarify next. In that case improved observations (in combination with improved analysis) will help fill the gap. Three main causes for the apparent gap can be identified:

(1) $\mathrm{S} / \mathrm{N}$ ratio limitations only allow the Stokes $V$ line ratio to be determined for $V$ amplitudes above $0.5 \%$. The intrinsic field strength $B_{\mathrm{f}}$ of the magnetic component decreases rather steeply as we go to small $V$ amplitudes. The lower cut-off of the strong-field branch at around $670 \mathrm{G}$ reflects the $B_{\mathrm{f}}$ value chosen in the limit of zero $V$, representing a linear extrapolation of the $B_{\mathrm{f}}$ curve across the $V$ interval $0.5-0 \%$. If we instead would assume that $B_{\mathrm{f}}$ decreases extremely steeply 
from 700 to zero $\mathrm{G}$ as we go from $V=0.5 \%$ to zero, then there would be no cut-off, and the gap would become populated. Future observations with better $\mathrm{S} / \mathrm{N}$ ratio may allow us to reliably determine the Stokes $V$ line ratio for smaller $V$ values to find out what actually happens rather than depending on extrapolations.

(2) The assumption that the small flux densities of the uncollapsed population represent fields that are so weak that we can equate flux density with field strength is likely to be very inadequate. If the fields are far from resolved, then the corresponding pixels harbor intrinsically much stronger fields. This may lead to a very substantial broadening of the inner, weak-field branch of the field-strength PDF, so that it spreads out to fill much of the gap. It is difficult to apply the $V$ line ratio to this flux population for two reasons: (i) the Stokes $V$ line ratio is insensitive to field strength and therefore nearly useless below about $400 \mathrm{G}$. (ii) The uncollapsed fluxes are very small and therefore more influenced by instrumental noise. For observational progress on both these issues we need improved $\mathrm{S} / \mathrm{N}$ ratio. In addition improved spatial resolution will enhance the visibility of the uncollapsed population to make it better distinguished from the collapsed population. Further, switching from the 6302/6301 to the $5250 / 5247$ line ratio would provide a more "pure" and reliable magnetic line ratio that is also more sensitive to smaller field strengths.

(3) From the previously discussed cancellation function in combination with the Hanle constraints we expect that most of the magnetic flux in the photosphere is invisible at the Hinode resolution, but will become increasingly visible as we improve the angular resolution. None of the current constraints prevents this hidden flux from having a wide field-strength distribution that may extend across the gap. Observations with increased resolution will help us uncover more of the hidden field and their intrinsic properties.

In summary, the situation concerning the magnetic dichotomy is the following: its reality as a property of the Sun is not in doubt for Stokes $V$ amplitudes in excess of $0.5 \%$, since we clearly have two distinctly separate populations of the $V$ line ratio, as seen for instance in Figs. 1 and 4. These two populations do indeed contribute to two disjunct PDF field strength branches, one weakfield and one strong-field branch, although the weak-field branch may be much wider than depicted in Fig. 16, since the line-ratio is too insensitive to constrain fields weaker than a few hundred $\mathrm{G}$. If we would simply exclude all weak polarization signals (below $0.5 \%$ ) by truncation of the inner, noise-affected part of the $V$ distribution, then we will certainly be left with a wide gap in the field strength PDF. However, the vast majority of all pixels belong to this inner part of the $V$ distribution. What is outside therefore cannot provide a representative sample of the PDF. We really need to include all the pixels that are too much affected by noise to give $V$ line ratios that are useful for determinations of intrinsic field strengths (although they still give useful vertical flux densities, since the effect of noise on the PDF for the apparent vertical flux densities is simply a Gaussian broadening of the PDF, which is harmless).

Here is the core of the problem. Since the $V$ region below $0.5 \%$ gives no useful information on intrinsic field strengths, we have included it by taking the functions that describe the lineratio behavior across the whole $V$ range $0.5-14 \%$ and extrapolated them across the small remaining $0.5 \%$ gap down to zero polarization. This extrapolation includes the function $r_{w}$, which is supposed to represent the relative abundance of the uncollapsed population (while the relative abundance of the collapsed population is $1-r_{w}$ ). Regardless of how we choose the extrapolated values of $r_{w}$, however, this whole procedure implicitly assumes that the magnetic dichotomy also exists in the $0-0.5 \%$ region, and what we need to do is only to assign relative weights to the two populations. This implicit assumption has the undesirable side effect that it effectively prevents any pixels from the $0-0.5 \%$ region to be assigned to the PDF gap region. Instead the procedure has the effect of merely redistributing these pixels between the separated weak-field and strong-field branches of the PDF.

What may instead happen is that the physical distinction between the two flux populations dissolves when approaching zero flux densities. The merging of the two populations would naturally happen across the region of intermediate field strengths, where the PDF gap is located. Since the majority of pixels represents this merger, they would suffice to completely fill the PDF gap, eliminate it, and connect the weak and strong fields in a continuous way.

While this appears to be a very plausible scenario that is not prohibited by any constraints, it remains a conjecture as long as it has not been proven. To move towards a proof we need observations that are better both in terms of $\mathrm{S} / \mathrm{N}$ ratio and angular resolution, to augment the domain in which the line ratio can be reliably determined. In addition we urgently need to switch to the more sensitive and "clean" 5250/5247 line ratio.

\section{Concluding remarks}

For vertical flux densities larger than about $30 \mathrm{G}$ we find from analysis of the Stokes $V$ line ratio a magnetic dichotomy with two flux populations, collapsed (representing $\mathrm{kG}$ type intermittent flux elements) and uncollapsed flux. They contribute to two disjunct regions of the PDF for the field strengths. Such a dualistic picture had been deduced before from the combination of Zeeman and Hanle observations, which led to the concern that it could be an artefact of using two mutually almost exclusive diagnostic tools. However, now the dichotomy is seen in Zeeman observations without any reference to the Hanle effect, and therefore cannot be a diagnostic artefact of this type.

The collapsed population is preferentially located in the intergranular lanes, while the uncollapsed population dominates in the bright cell interiors. A similar correlation has been found for the "hidden", spatially unresolved flux of mixed polarities from an analysis of the Hanle depolarization effect in molecu$\operatorname{lar}\left(\mathrm{C}_{2}\right)$ and atomic (Sr I) lines by Trujillo Bueno et al. (2004) by using polarized radiative transfer in a $3 \mathrm{D}$ model atmosphere generated by numerical simulation. Using a model with an isotropic, single-valued field they find a field strength of order $10 \mathrm{G}$ for the $\mathrm{C}_{2}$ line but $60 \mathrm{G}$ for the $\mathrm{Sr}$ line. Since the model calculations show that the $\mathrm{C}_{2}$ line is only abundant in the bright cell interiors while the $\mathrm{Sr}$ line does not exhibit any strong correlation with the solar granulation, these results indicate that much more flux is hidden in the intergranular lanes than in the cell interiors and is therefore being shuffled around as the granulation pattern evolves. This interpretation has recently been supported by Snik et al. (2010), who studied how the scattering polarization in the $\mathrm{CN}$ molecular band correlates with the solar granulation. They found significantly reduced scattering polarization in the intergranular regions, indicating more Hanle depolarization and stronger "hidden" fields there, although a more definite interpretation would require detailed polarized $\mathrm{CN}$ line synthesis with a realistic MHD model.

The Hanle $60 \mathrm{G}$ result, when compared with the average of the total flux density of about $20 \mathrm{G}$ in the Hinode quiet-Sun data, 
indicates that most of the magnetic flux remains invisible at the Hinode resolution scale due to cancellation of opposite polarities within the resolution element. A scaling law for the average unsigned flux density, the cancellation function, indicates how the flux visibility will improve with increased resolution. The power-law fit to this function as determined in the resolved domain can be extrapolated into the unresolved domain under the implicit assumption of self-similarity for a fractal-like field, which may not be valid. Our analysis results in a cancellation function that is much less steep than that of Pietarila Graham et al. (2009), with a cancellation exponent that is only half of theirs. We suspect that the discrepancy is due to the circumstance that they implicitly give more weight to the inner region of the PDF by not correcting for the line weakenings of the collapsed population in their data conversion to flux densities. The inner PDF region is likely to contain much more of locally mixed polarities than the PDF tails, which are represented by rather isolated strong-field concentrations. Since the hidden fields that will be increasingly revealed with improving angular resolution are likely to be physically more related to the inner part of the PDF, it may well be that the cancellation function steepens as we go to smaller scales, and that therefore the relative importance of the mixed-polarity fields increases. While this may be a non-fractal behavior, we know of no physical arguments that would force the field to comply with the rules of a given fractal over a large range of scales.

Due to the large amount of missing flux indicated by the Hanle analysis we need to extrapolate the cancellation function over scales differing by orders of magnitude, regardless of the detailed behavior of the cancellation exponent. It thus seems likely that much of the hidden flux resides at scales that are close to the end of the magnetic scale spectrum, where the diffusive time scale becomes shorter than the convective time scale. This scale is estimated to be near $10 \mathrm{~m}$ if we use the Spitzer conductivity. While the Spitzer formula may not be adequate in the upper photosphere where the hydrogen ionization is low, it is probably useful in the region just below the photosphere, which is the most relevant region for structure formation by magnetic turbulence.

From the comparison between flux density and intrinsic field strength (as derived from the line ratio of the collapsed population) we can derive the sizes of the strong-field elements or flux tubes and their probability distributions. The majority of the flux tubes have sizes in the range $10-70 \mathrm{~km}$, which satisfies the requirements for laterally optically thick flux tubes in the layers just below the photosphere, where the superadiabatic gradient has a maximum and the conditions for convective collapse are optimum. The derived intrinsic field strengths decrease rather steeply with decreasing size and become smaller than $\mathrm{kG}$ for sizes below about $60 \mathrm{~km}$. Instrumental noise prevents a good determination of the intrinsic field strength below the $60 \mathrm{~km}$ size, but the trend indicates that when we reach scales of order $10 \mathrm{~km}$ the field may be more like hectogauss. The concept of intermittent flux tubes then dissolves and may need to be abandoned. At such scales and below convective collapse ceases to be efficient. We are then left with a field that is amplified and shredded by the turbulent convection, and which represents the hidden flux that is demanded by the Hanle observations.

Acknowledgements. I want to acknowledge the fruitful, in-depth discussions about these issues that took place at ISSI (International Space Science Institute) in Bern during a meeting with an ISSI International Team, November 15-19, 2010. I also want to thank Jonathan Pietarila Graham for illuminating discussions concerning the aspect ratios of smoothing windows for the derivation of cancellation functions, and Javier Trujillo Bueno for clarifying the role of 3D model atmospheres for the interpretation of the Hanle depolarization. The analysis has been based on observations done by the Hinode satellite. Hinode is a Japanese mission developed and launched by ISAS/JAXA, with NAOJ as domestic partner and NASA and STFC (UK) as international partners. It is operated by these agencies in co-operation with ESA and NSC (Norway).

\section{References}

Bommier, V., \& Molodij, G. 2002, A\&A, 381, 241

Danilovic, S., Schüssler, M., \& Solanki, S. K. 2010, A\&A, 509, A76

Fischer, C. E., de Wijn, A. G., Centeno, R., Lites, B. W., \& Keller, C. U. 2009, A\&A, 504, 583

Frazier, E. N., \& Stenflo, J. O. 1972, Sol. Phys., 27, 330

Howard, R., \& Stenflo, J. O. 1972, Sol. Phys., 22, 402

Kosugi, T., Matsuzaki, K., Sakao, T., et al. 2007, Sol. Phys., 243, 3

Lites, B. W., Kubo, M., Socas-Navarro, H., et al. 2008, ApJ, 672, 1237

Maltby, P., Avrett, E. H., Carlsson, M., et al. 1986, ApJ, 306, 284

Nagata, S., Tsuneta, S., Suematsu, Y., et al. 2008, ApJ, 677, L145

Orozco Suárez, D., Bellot Rubio, L. R., del Toro Iniesta, J. C., et al. 2007, ApJ, 670, L61

Parker, E. N. 1978, ApJ, 221, 368

Pietarila Graham, J., Danilovic, S., \& Schüssler, M. 2009, ApJ, 693, 1728

Schüssler, M., \& Vögler, A. 2008, A\&A, 481, L5

Snik, F., de Wijn, A. G., Ichimoto, K., et al. 2010, A\&A, 519, A18

Spruit, H. C. 1977, Magnetic flux tubes and transport of heat in the convection zone of the sun (Utrecht)

Spruit, H. C. 1979, Sol. Phys., 61, 363

Spruit, H. C., \& Zweibel, E. G. 1979, Sol. Phys., 62, 15

Stein, R. F., \& Nordlund, A. 2006, ApJ, 642, 1246

Stenflo, J. O. 1971, in Solar Magnetic Fields, ed. R. Howard, IAU Symp., 43, 101

Stenflo, J. O. 1973, Sol. Phys., 32, 41

Stenflo, J. O. 1982, Sol. Phys., 80, 209

Stenflo, J. O. 1987, Sol. Phys., 114, 1

Stenflo, J. O. 1994, Solar Magnetic Fields - Polarized Radiation Diagnostics (Kluwer)

Stenflo, J. O. 2010, A\&A, 517, A37

Stenflo, J. O., Solanki, S., Harvey, J. W., \& Brault, J. W. 1984, A\&A, 131, 333

Stenflo, J. O., Bianda, M., Keller, C. U., \& Solanki, S. K. 1997, A\&A, 322, 985

Suematsu, Y., Tsuneta, S., Ichimoto, K., et al. 2008, Sol. Phys., 249, 197

Trujillo Bueno, J., Collados, M., Paletou, F., \& Molodij, G. 2001, in Advanced Solar Polarimetry - Theory, Observation, and Instrumentation, ed. M. Sigwarth, ASP Conf. Ser., 236, 141

Trujillo Bueno, J., Shchukina, N., \& Asensio Ramos, A. 2004, Nature, 430, 326

Tsuneta, S., Ichimoto, K., Katsukawa, Y., et al. 2008, Sol. Phys., 249, 167

Unno, W., \& Ando, H. 1979, Geophys. Astrophys. Fluid Dyn., 12, 107 


\section{Appendix A: Pitfalls with the horizontal field}

In recent years there has been a controversy concerning the role and importance of the horizontal magnetic fields on the quiet Sun. Starting with the Hinode analysis of Orozco Suárez et al. (2007) and Lites et al. (2008) there have been frequently repeated claims that there is about 5 times larger horizontal than vertical flux density on the quiet Sun. In contrast an in-depth analysis of the identical Hinode data set by Stenflo (2010) finds that the angular distribution of field vectors is strongly peaked around the vertical direction for large flux densities, while the distribution widens as the flux density decreases to become isotropic in the limit of zero flux density. The conclusion is that there is no evidence for any preponderance of horizontal fields.

Such discrepancies can arise since it is vastly more difficult to diagnose transversal than longitudinal magnetic fields. In the following our discussion will refer to the disk center, where the horizontal fields are transversal, while the vertical fields are longitudinal, i.e., directed along the line of sight. One reason for the difference in difficulty is the low sensitivity of the linear polarization to weak magnetic fields as compared with the circular polarization. Thus the same polarization noise, when translated to flux densities, is larger for the horizontal fields by a factor of order 25. Another difficulty is that spatially unresolved substructures that are improperly accounted for lead to much larger errors in the determined horizontal fields than in the vertical ones. In this Appendix we will try to elucidate these pitfalls.

\section{A.1. Meaning of flux densities and filling factors for the horizontal fields}

Let us first try to clear up some confusion in the use of the concepts flux density and filling factor for the horizontal fields. Our use here of the concept "flux density" is equivalent to "average field strength", meaning magnetic flux divided by the area of the resolution element. Since the term "resolution element" appears to refer to the 2D angular resolution element perpendicular to the line of sight, confusion often arises in the understanding of the term flux density for the transverse field, since this field component lies in the plane of the angular resolution element. However, we have to remember that the actual resolution element is a $3 \mathrm{D}$ volume, namely the area of the angular resolution element times the depth of spectrum formation along the line of sight. The shape of this volume element does not matter, we can imagine magnetic flux penetrating it in any direction, unrelated to the direction of the line of sight, and define a flux density (or average field strength) for the field component along any such direction. Flux density in the horizontal direction is therefore as well-defined a concept as flux density in the vertical direction.

A similar confusion has arisen around the use of filling factors for the horizontal field, with misled suggestions that one may need to use different filling factors for the horizontal and vertical fields. The source of this confusion again has to do with the distinction between 2D and 3D resolution elements. The magnetic filling factor is often defined as the fraction of the area (of the angular resolution element) that is occupied by the magnetic component, causing confusion as to what this area should be in the case of the horizontal field. This confusion is however a result of a misunderstanding of what filling factor means. To avoid such confusion it should always be seen as a volume filling factor (fraction of the 3D resolution element occupied by the magnetic component), never as an area filling factor. In the special case of vertical flux tubes the concepts of volume filling factor and area filling factor become identical, but in the general case (inclined fields) only the volume filling factor is a meaningful concept. It would be completely unphysical to use separate filling factors for vertical and horizontal fields. Whatever orientation a magnetic component has inside the volume resolution element, the occupation fraction of the volume must be identical for all field components, horizontal as well as vertical.

\section{A.2. Apparent predominance of horizontal fields for an isotropic angular distribution}

Another misleading habit in previous literature has been to form the ratio $B_{\perp} / B_{\|}$and implicitly suggest incorrectly that when this ratio exceeds unity, then the field is preferentially horizontal rather than vertical. However, it is only meaningful to speak of a preference for horizontal fields if the angular distribution of the field vectors is flatter than an isotropic distribution, which is spherical. As explained in Stenflo (2010) the ratio $B_{\perp} / B_{\|}$is not unity for an isotropic distribution, but $\pi / 2 \approx 1.57$. This is so because the horizontal field is $B \sin \gamma$, the vertical field $B \cos \gamma$, and the ratio between the angular averages of $\sin \gamma$ and $\cos \gamma$ is $\pi / 2$. The reason for this artificial advantage of the horizontal field is that it is defined as the projection of the field vector onto a plane, while the vertical field is defined as the projection onto an axis. Since one has to compare the $B_{\perp} / B_{\|}$ratio not with unity but with 1.57 , the published ratio of about 5 implies a horizontal dominance with respect to the isotropic case by approximately a factor of 3, not a factor of 5. Still, as shown in Stenflo (2010), this remaining factor of 3 is much too high, and there is no evidence from the Hinode data that it exceeds unity. Next we will discuss the two main pitfalls that can easily lead to large spurious factors: the profoundly different ways in which noise and the filling factor affect the horizontal and vertical fields.

\section{A.3. PDF distributions of measurement noise}

In the weak-field limit (below a few hundred $\mathrm{G}$, where the Zeeman splitting is still small in comparison with the line width) the following proportionalities apply in the relations between polarization and field strength:

$\begin{aligned} B_{\|, \text {app }} & \sim V_{\text {obs }}, \\ B_{\perp, \text { app }} & \sim\left[Q_{\mathrm{obs}}^{2}+U_{\mathrm{obs}}^{2}\right]^{1 / 4},\end{aligned}$

where we have added index notations "obs" and "app" to cover the case when the field is not resolved and we have a filling factor $f$, so that

$Q_{\mathrm{obs}}=f Q$

and similarly for $U$ and $V$. If the "true" filling factor were unity, then the apparent field $B_{\perp \text {,app }}$ would equal the corresponding "true" field $B_{\perp}$. For a given filling factor $f$ the average flux density is

$\left\langle B_{\perp}\right\rangle=f B_{\perp}$,

but it does not equal $B_{\perp \text {, app }}$ (see below). In contrast

$\left\langle B_{\|}\right\rangle=f B_{\|}=B_{\|, \text {app }}$.

To quantitatively explore the influence of noise on the determination of weak magnetic fields we next assume a filling factor of unity (since this was also done in the analysis of Lites et al. 2008) and use the detailed calibrations in Figs. 19 and 21 of Stenflo (2010) that are representative of the uncollapsed flux population, in order to find the constants of proportionality in Eq. (A.1). If 


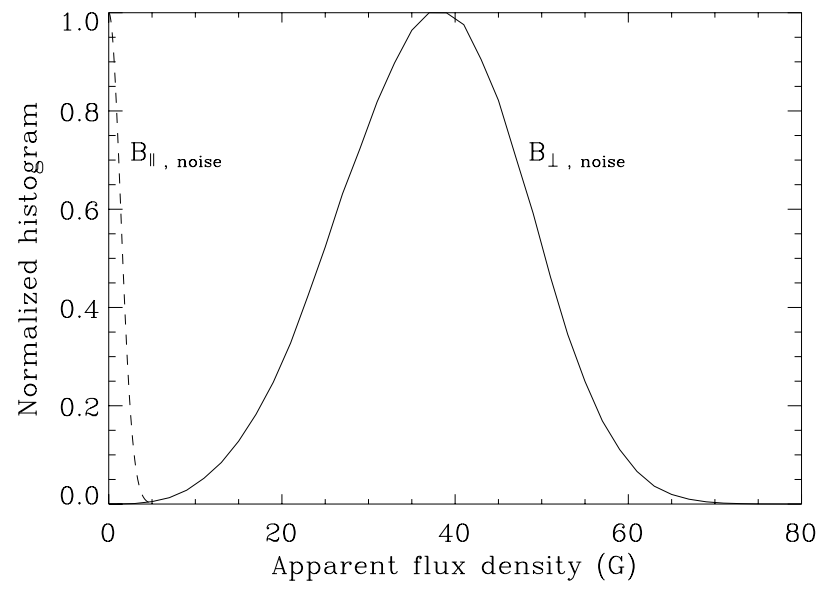

Fig. A.1. Histograms of the apparent flux densities for the horizontal field $B_{\perp, \text { app }}$ (solid curve) and the vertical field $B_{\|, \text {app }}$ (dashed curve), derived from the Gaussian polarization noise with standard deviations $0.035 \%$ in $Q$ and $U$ and $0.047 \%$ in $V$ (in units of the continuum intensity).

the polarizations $Q, U, V$ are expressed in $\%$ of the continuum intensity and the field strength in $\mathrm{G}$, then the constant of proportionality is 29.4 in the first equation and 184 in the second.

The polarization noise distributions in $Q, U$, and $V$ are Gaussian. Since we have direct proportionality between $B_{\|}$and $V$, the noise distribution in $B_{\|}$is Gaussian as well. In contrast the noise distribution in $B_{\perp}$ is entirely different, due to the very non-linear relation between polarization and field strength.

The measured standard deviations (1- $\sigma$ errors) in \% of the continuum intensity for the Gaussian noise distributions in the measured values of the polarization amplitudes in the Hinode SP deep mode data set are $0.035 \%$ for each of $Q$ and $U$, and slightly worse, $0.047 \%$, for $V$. These low-noise values are testimony for the excellent quality of the Hinode spectro-polarimeter. With Eq. (A.1) and their respective calibration factors these small polarizations translate into $1.37 \mathrm{G}$ in the case of Stokes $V$ and to the 25 times larger value of $34.5 \mathrm{G}$ for Stokes $Q$ or $U$.

With the known Gaussian distributions for $Q, U$ and $V$ and with Eq. (A.1) we have used Monte Carlo simulations to derive how the noise propagates into $B_{\perp}$. The result is shown in Fig. A.1. The noise distribution for $B_{\perp}$ spreads between 5 and $70 \mathrm{G}$. Due to the noise there will not be any apparent horizontal fields weaker than 5 G, while the analysis in Stenflo (2010) shows that the great majority of pixels have vertical flux densities that are smaller than $5 \mathrm{G}$. If horizontal flux densities that are only modestly affected by noise are incorrectly assumed to be solar and are combined with the many small vertical flux density values, then we immediately obtain a spurious picture where the horizontal fields appear to dominate. It does not take much of a residual of the noise ratio of 25 between the horizontal and vertical fields to infiltrate the analysis to account for the factor of 3 that has been claimed to represent the predominance of the horizontal fields.

\section{A.4. Influence of incorrect filling factors for the horizontal fields}

The second potential pitfall that would always work in the direction of spuriously enhancing the $\left\langle B_{\perp}\right\rangle /\left\langle B_{\|}\right\rangle$ratio is to use too large a value for the filling factor. As seen from Eqs. (A.1)-(A.4)

$$
\frac{B_{\perp, \text { app }}}{B_{\|, \text {app }}} \sim \frac{1}{\sqrt{f}} \frac{\left\langle B_{\perp}\right\rangle}{\left\langle B_{\|}\right\rangle}
$$

In their analysis Lites et al. (2008) assumed a value of unity for $f$. If this assumption is incorrect, then the derived ratio between the horizontal and vertical flux densities is too large by a factor of $1 / \sqrt{f}$. If for instance the "true" filling factor is $10 \%$, then the average horizontal flux density is spuriously enhanced by more than a factor of 3 , enough to account for the previously claimed predominance of the horizontal fields. As we have seen in Sect. 5, the great majority of pixels that represent the collapsed flux population have filling factors smaller than $10 \%$. This illustrates that the filling factor effect alone can fully account for the factor of 3 discrepancy in the literature.

While the claim that there is a predominance of horizontal flux is based on the use by Lites et al. (2008) of filling factor unity at disk center, they also tried to apply Milne-Eddington inversions to determine the actual values of the filling factors. Since such inversions can only be used for observations with sufficient $\mathrm{S} / \mathrm{N}$ ratio, they could only apply it to $1 / 3$ of the pixels, which are representative of the strongest flux concentrations. They found that for this sample of pixels the typical filling factor was about $20 \%$. This implies that their discrepancy factor of 3 (favoring horizontal fields with respect to an isotropic distribution) would get changed to $3 \times \sqrt{0.2} \approx 1.3$, which is quite close to the value of 1.0 for the isotropic case. As we find (from our own analysis) that the majority of the $2 / 3$ of the small-flux pixels that could not be analysed by inversion have substantially smaller filling factors, the remaining factor of 1.3 can easily be accounted for by the filling-factor effect alone. For this reason one can say that the Lites et al. (2008) analysis does not lead to any evidence that there is a preponderance of horizontal fields, in contrast to the widespread belief that is based on that paper.

\section{A.5. Empirical tests near the Sun's limb}

In summary we have seen how a small infiltration of noise or an incorrectly assumed filling factor of unity will both artificially enhance the average horizontal flux density with respect to the average vertical flux density. Each effect on its own can easily account for the claimed preponderance of the horizontal fields by the factor of 3 , but the combination of both makes the effect potentially much larger.

For those who still believe that there is a preponderance of horizontal fields, there is a straightforward test that may empirically settle the matter, namely to apply the same analysis technique to Hinode data near the solar limb. If we define the Stokes $Q$ direction to be parallel to the radius vector, then near the limb the longitudinal Zeeman signatures in Stokes $V$ diagnose the horizontal component, while the transverse Zeeman signatures of opposite signs in Stokes $Q$ diagnose both the vertical component and the horizontal component that is parallel to the limb.

The best test, which does not depend on any comparison between horizontal and vertical fields, is to compare the apparent magnitudes of the two horizontal components, the one diagnosed by Stokes $Q$ (in the transversal plane) and the one diagnosed by Stokes $V$ (along the line of sight). Statistically these components must be of the same magnitudes on the quiet Sun. It would be totally unphysical if there would be a preference for the transversal plane, since the Sun does not care where the observer is located. If one would find that the apparent field in the transversal plane is systematically stronger, then the effects that we have discussed have infiltrated the analysis and made it faulty.

Limb observations that allow such a test were indeed presented in the paper of Lites et al. (2008). They used a deep mode 
Hinode recording of March 15, 2007, with the spectrograph slit crossing the south polar limb. Their results (in their Figs. 15 and 17) clearly show that there is always a huge preponderance of the apparent magnetic flux in the transversal plane as compared with the line-of-sight magnetic flux, for all center-to-limb distances, all the way to the limb. This systematic predominance of the transversal plane can hardly be a property of the Sun, since near the limb the transverse plane is perpendicular to the horizontal plane.

This test can easily be applied to any data set that includes data near the limb and claims to measure the transverse magnetic fields on the quiet Sun correctly. For instance, it could be used to test the data reduction pipeline for HMI on the solar dynamics observatory (SDO) or for SOLIS. 